\title{
Preferential Belief Change Using Generalized Epistemic Entrenchment
}

\author{
HANS ROTT \\ Fachgruppe Philosophie, Universität Konstanz, Postfach 5560 , \\ D-7750 Konstanz, Germany \\ email:pirctt@dknkurz1.bitnet
}

(Received 1 March, 1991; in final form 3 December, 1991)

\begin{abstract}
A sentence $A$ is epistemically less entrenched in a belief state $K$ than a sentence $B$ if and only if a person in belief state $K$ who is forced to give up either $A$ or $B$ will give up $A$ and hold on to $B$. This is the fundamental idea of epistemic entrenchment as introduced by Gärdenfors (1988) and elaborated by Gärdenfors and Makinson (1988). Another distinguishing feature of relations of epistemic entrenchment is that they permit particularly simple and elegant construction recipes for minimal changes of belief states. These relations, however, are required to satisfy rather demanding conditions. In the present paper we liberalize the concept of epistemic entrenchment by removing connectivity, minimality and maximality conditions. Correspondingly, we achieve a liberalization of the concept of rational belief change that does no longer presuppose the postulates of success and rational monotony. We show that the central results of Gärdenfors and Makinson are preserved in our more flexible setting. Moreover, the generalized concept of epistemic entrenchment turns out to be applicable also to relational and iterated belief changes.
\end{abstract}

Key words: Belief revision, epistemic entrenchment, theory change.

\section{INTRODUCTION}

A sentence $A$ is epistemically less entrenched in a belief state $K$ than a sentence $B$ if and only if a person in belief state $K$ who is forced to give up either $A$ or $B$ will give up $A$ and hold on to $B$. This is the fundamental idea of epistemic entrenchment as introduced by Peter Gärdenfors (see Gärdenfors 1988 and Gärdenfors and Makinson 1988). It emerged that relations of epistemic entrenchment permit a very simple and elegant construction recipe for rational changes of belief states (see the definitions (Def - ) and (Def $*$ ) below). I think it is fair to make the following terminological decision. A binary relation between sentences of a given language is a relation of epistemic entrenchment only if it conforms to the fundamental intuitive idea and permits a very simple formal construction of belief changes.

The current theory of epistemic entrenchment has many virtues and a number of drawbacks. With epistemic entrenchment one can do anything which can be done by other established methods of belief change - provided one accepts the entire set of the so-called Gärdenfors postulates for belief

Journal of Logic, Language and Information 1: 45-78, 1992.

(C) 1992 Kluwer Academic Publishers. Printed in the Netherlands. 
revision (see Gärdenfors 1988, Gärdenfors and Makinson 1988, Rott 1991a, 1992). But there is no flexibility of accepting and rejecting Gärdenfors's supplementary postulates as it is in partial meet and safe contraction (see Alchourrón, Gärdenfors and Makinson 1985, Alchourrón and Makinson 1985). And there are three formal restrictions for epistemic entrenchment which one would intuitively like to remove. In this paper, I will generalize the approach of Gärdenfors and Makinson (1988), with inspiration drawn from Makinson (1989, 1990), Kraus, Lehmann and Magidor (1990), and Makinson and Gärdenfors (1991).

The liberalization of epistemic entrenchment will consist in the removal of three formal restrictions. We will have no top, bottom and connectivity conditions. An alternative suggestion to remove connectivity from the requirements for epistemic entrenchment is due to Lindström und Rabinowicz (1991). But as they have to resort to a fairly complicated construction recipe for belief changes, their account fails to specify a relation of epistemic entrenchment in the sense of our terminological decision. We will be left with no theory-relative conditions, nor will we employ non-Horn conditions. We call our relation a relation of generalized epistemic entrenchement (GEErelation) and contrast it with the relation of standard epistemic entrenchment (SEE-relation) of Gärdenfors and Makinson.

The usual dependency between theories and relations of epistemic entrenchment may be regarded as reversed. While Gärdenfors and Makinson have SEE-relations with respect to theories, we will define theories with respect to GEE-relations. In this setting, the modelling of iterated belief change is possible, and we will see that one single GEE-relation suffices to define a whole belief contraction model. A GEE-relation contains more information than a (preferential) contraction function over a consistent theory $K$; it contains exactly the same information as a (preferential) contraction function over the inconsistent theory $L$.

By liberalizing epistemic entrenchment we also liberalize the corresponding logic of theory change, nonmonotonic logic, or conditional logic. First, we remove Gärdenfors's basic condition $(\mathrm{K}-4)$ and allow that a reasoner refuses to withdraw sentences which are no logical truths. He or she may have a protected "hard core" of sentences which survive every conceivable change of belief. Secondly, we find that both of Gärdenfors's supplementary conditions are doing two jobs at a time: $(\mathrm{K} \div 7)$, or rather its analogue $(\mathrm{K} * 7)$ for belief revisions, implies "cut" and "distributivity", while $(\mathrm{K}-8)$, or rather its analogue $(\mathrm{K} * 8)$ for belief revisions, implies "cautious monotony" and "rational monotony" (for the meaning of these terms, see Kraus, Lehmann and Magidor 1990, Lehmann and Magidor 1990, and Makinson 1990). The most striking feature of our treatment is that we will do without rational monotony. 
In belief revision, $(\mathrm{K}-8)$ is the first condition to be lost when looking at some more general method of partial meet contraction or safe contraction (see Alchourrón, Gärdenfors and Makinson 1985, and Alchourrón and Makinson 1985). In nonmonotonic logic, David Makinson (1990) for instance is quite hesitant to accept rational monotony as a desirable property, and he shows that it is not satisfied by virtually all of the standard explications of defeasible reasoning. A corresponding axiom scheme in conditional logic has been criticized by Pollock and Ginsberg. Thus it seems desirable to be able to do away with rational monotony while still working with a genuine relation of epistemic entrenchment. In analogy to the terminology coined by Kraus, Lehmann and Magidor (1990), we will talk of preferential belief change in this paper.

The object language under consideration is supposed to include the language of propositional logic. It contains the propositional operators $\neg, \wedge, \vee$, $\rightarrow$ and $\leftrightarrow$ (while $\&, \Rightarrow$ and $\Leftrightarrow$ are used metalinguistically). By $L$ we denote the set of all sentences of this language. In Section 6 we presume that $L$ is countable. Throughout the whole paper, variables $A, B, C$, etc. range over sentences in $L, H$ over sets of sentences in $L$, and $K, K^{\prime}$, etc. over theories in $L$, i.e., sets of sentences in $L$ which are closed under some (well-behaved) underlying consequence operation $C n$. We assume that $C n$ includes tautological implication, is compact and satisfies the deduction theorem. We use $C n(A)$ to denote $C n(\{A\})$, and $H+A$ as an abbreviation of $C n(H \cup\{A\})$. We also use $H \vdash A$ for $A \in C n(H)$.

\section{EPISTEMIC ENTRENCHMENT:}

\section{THE STANDARD THEORY OF GÄRDENFORS AND MAKINSON}

The basic problem is how to eliminate sentences from a given belief state $K$ under certain integrity constraints. We recapitulate the theory of belief contraction and epistemic entrenchment as developed by Gärdenfors and Makinson (Gärdenfors 1988 and Gärdenfors and Makinson 1988) without comments on the intuitive content of the postulates. The reader is referred to the original work for motivation and further information.

Postulates for Gärdenfors contractions (over $K$ )

$(\mathrm{K} \dot{-1}) K \dot{-} A$ is a theory

$(\mathrm{K} \dot{2}) \mathrm{L}) \cdot A \subseteq K$

$(\mathrm{K}-3)$ If $A \notin K$ then $K \dot{-} A=K$

$(\mathrm{K} \dot{-4)}$ If $A \in K \dot{-} A$ then $\vdash A$

$(\mathrm{K} \dot{\circ}) K \subseteq(K \dot{-} A)+A$

$(\mathrm{K} \dot{-6})$ If $C n(A)=C n(B)$ then $K \dot{-} A=K \dot{-} B$ 
$(\mathrm{K} \div 7) K \dot{-} A \cap K \dot{-} B \subseteq K \dot{-} \wedge \wedge B$

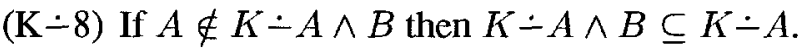

$(\mathrm{K} \bullet 1)-(\mathrm{K}-6)$ are Gärdenfors's basic postulates, $(\mathrm{K}-7)$ and $(\mathrm{K}-8)$ are the supplementary ones. $(\mathrm{K}-1),(\mathrm{K} \div 5),(\mathrm{K}-7)$ and $(\mathrm{K}-8)$ are restricted to the case where $K$ is a theory. We do not explicitly mention this, because in the present paper we consider only the case where $K$ is in fact a theory or, as is common jargon, a belief set. At least $(\mathrm{K}-8)$, as well as its counterparts in conditional and nonmonotonic logic, are not universally accepted.

Postulates for standard epistemic entrenchment (with respect to $K$ )

(SEE1) If $A \leq B$ and $B \leq \mathrm{C}$ then $A \leq \mathrm{C}$

(Transitivity)

(SEE2) If $A \vdash B$ then $A \leq B$

(Dominance)

(SEE3) $A \leq A \wedge B$ or $B \leq A \wedge B$

(Conjunctiveness)

(SEE4) If $K \neq L$ then: $A \leq B$ for every $B$ iff $A \notin K$

(Bottom)

(SEE5) If $B \leq A$ for every $B$ then $\vdash A$

If $\leq$ is a SEE-relation with respect to a belief set $K$ then " $A \leq B$ " means that $B$ is at least as firmly entrenched in $K$ as $A$. So far we have only introduced postulates for infinite entities, but it is not difficult to offer finite representations of an important class of SEE-relations (see Rott 1991b, Section 3). The following facts are intuitively unpleasant:

- because connectivity follows from (SEE1) - (SEE3), it is forbidden to stay undecided in the comparison of two arbitrary sentences $A$ and $B$;

- because of (SEE4), it is forbidden to have a gradation of the sentences which are not included in the current belief set $K$;

- because of (SEE5), it is forbidden to assign to some sentence a degree of epistemic entrenchment which is as high as the degree of logical truth.

From contractions to standard epistemic entrenchment

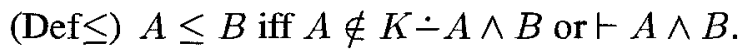

In order to discard $A \wedge B$ from a belief set $K$ it is both necessary and sufficient to discard either $A$ or $B$ (or both) from $K$. So $A \leq B$ may be interpreted tentatively as saying that when it comes to deciding whether to give up either $A$ or $B$, the reasoner with epistemic entrenchment relation $\leq$ is ready to give up $A$. The SEE-relation $\leq$ with respect to $K$ defined from a given contraction function $\dot{-}$ over $K$ by $(\operatorname{Def} \leq)$ is denoted $\mathbf{E}(\dot{-})$.

From standard epistemic entrenchment to contractions
$(\mathrm{Def}-) \quad K \dot{-} A=\left\{\begin{array}{l}K \cap\{B: A<A \vee B\} \\ K\end{array}\right.$
if $\forall A$
otherwise 
Here $A<A \vee B$ means $A \leq A \vee B$ and $A \vee B \not \leq A$. By connectivity, this is equivalent to $A \vee B \not \leq A$. The contraction function - over $K$ defined from a given SEE-relation $\leq$ with respect to $K$ by (Def $\dot{-}$ ) is denoted $\mathbf{C}(\leq)$. A good deal of justification of this definition derives from the plausibility of $($ Def $\leq$ ) and the following

\section{Theorem 1 (Gärdenfors and Makinson 1988)}

(i) If $\leq$ satisfies (SEE1) - (SEE5) then $\mathrm{C}(\leq)$ satisfies $(\mathrm{K}-1)-(\mathrm{K} \dot{-8})$.

(ii) If - satisfies $(\mathrm{K}-1)-(\mathrm{K}-8)$ then $\mathbf{E}(\dot{-})$ satisfies (SEE1) - (SEE5).

(iii) If $\leq$ satisfies $($ SEE1) $-($ SEE5) then $\mathbf{E}(\mathbf{C}(\leq))=\leq$.

(iv) If $\dot{-}$ satisfies $(\mathrm{K} \dot{-} 1)-(\mathrm{K} \dot{-})$ then $\mathbf{C}(\mathbf{E}(\dot{-}))=\dot{-}$.

Bearing in mind the above-mentioned points of critique, it is natural to ask whether these very nice results can be reproduced in a more general setting.

\section{REVISIONS AND CONTRACTIONS}

Philosophically, contractions are more basic than revisions. In order to minimally revise a belief set $K$ by a sentence $A$, first make $K$ consistent with $A$, i.e., remove $\neg A$ in some rational and economical way, then add $A$, and finally close under $C n$. Most writers in belief revision have advocated this strategy. One of the first and most vigorous proponents was Isaac Levi.

(Levi identity) $K * A=(K \dot{-} \neg A)+A$.

Now consider the following list of "translations" of some eminent patterns of nonmonotonic reasoning into the language of belief revision. We mention unit versions of cut, cautious monotony, distribution, the hard half of the deduction theorem ("conditionalization"), and rational monotony (see Kraus, Lehmann and Magidor 1990, Makinson 1990, and Makinson and Gärdenfors 1991).

(Cut) If $B \in K * A$ then $K * A \wedge B \subseteq K * A$.

(CM) If $B \in K * A$ then $K * A \subseteq K * A \wedge B$.

(Dis) $\quad K * A \cap K * B \subseteq K * A \vee B$.

(HHD) $K * A \wedge B \subseteq(K * A)+B$.

(RM) If $\neg B \notin K * A$ then $(K * A)+B \subseteq K * A \wedge B$.

In the presence of the basic Gärdenfors postulates for revision, (Dis) is equivalent to (HHD) (see Gärdenfors 1988, pp. 56-57). It is remarkable and surprising that neither (Cut) nor (CM) is derivable from the basic Gärdenfors postulates alone. Given the basic postulates, however, $(\mathrm{K} * 7)$, which is (HHD) (or equivalently, which is (Dis)), implies Cut, and $(\mathrm{K} * 8)$, which is (RM), 
implies Cautious Monotony (see Makinson and Gärdenfors 1991). What are the corresponding postulates for contractions obtained via the Levi identity?

Lemma 1 Given the Levi identity and the basic postulates for contractions, the following conditions for - are equivalent with (Cut), (CM), (Dis) and (RM), respectively:

$(\mathrm{K} \div \mathrm{7c})$ If $B \in K \dot{-} \wedge \wedge B$ then $K \dot{-} A \subseteq K \dot{-} A \wedge B$.

$(\mathrm{K} \dot{\circ} 8 \mathrm{c})$ If $B \in K \dot{-} \wedge \wedge$ then $K \dot{-} \wedge \wedge B \subseteq K \dot{-}$.

$(\mathrm{K} \dot{-7)} \quad K \dot{-} A \cap K \dot{-} B \subseteq K \dot{-} A \wedge B$

$(\mathrm{K} \div 8)$ If $A \notin K \dot{-} A \wedge B$ then $K \dot{-} A \wedge B \subseteq K \dot{-}$.

Given $(\mathrm{K} \dot{-1})-(\mathrm{K} \dot{-6}),(\mathrm{K} \dot{-7})$ implies $(\mathrm{K} \dot{-7} \mathrm{c})$ and $(\mathrm{K} \dot{-8})$ implies $(\mathrm{K}-8 \mathrm{c})$.

Proofs of the lemmas and theorems are collected in an appendix at the end of the paper.

The obvious question is whether one can have the contraction counterparts of (Cut) and (CM) - which represent two of the most basic patterns of nonmonotonic reasoning - without having the full power of (HHD) and (RM), respectively. Before answering this question we turn to a trivial formal exercise with nontrivial intuitive consequences.

\section{RELATIONS}

Research in partial meet contractions and epistemic entrechment contractions has been done by studying non-strict (reflexive) relations $\leq$ (see Alchourrón, Gärdenfors and Makinson 1985, Gärdenfors 1988, Gärdenfors and Makinson 1988). It turned out that transitivity conditions in this context are both very powerful and problematic, while connectivity conditions are neither powerful nor problematic (see Alchourrón, Gärdenfors and Makinson 1985, pp. 519524, Gärdenfors and Makinson 1988, proof of Theorem 5). Research in safe contractions, on the other hand, has focused on a strict (irreflexive) relation $<$ (see Alchourrón and Makinson 1985, 1986, Rott 1992). Here the situation is just the reverse. Transitivity is harmless and indeed not even needed for many purposes, while "virtual connectivity" is very powerful. Intuitively, the case of the strict relations is the right one. In general one is willing to accept transitivity as a very natural requirement, while connectivity conditions put a great strain on a realistic image of a reasoner. Why shouldn't there be incomparabilities? We can resolve this tension by regarding the strict relation $<$ as primary and interpreting the non-strict $\leq$ as its converse complement. This will turn incomparabilities under $<$ into indifferences under $\leq$. Reinterpreting $\leq$ in this way is, to be sure, somewhat misleading philosophically, but it helps the various formal properties of the relations in point to get their due intuitive places. Connectivity of $\leq$ is then natural, transitivity is not. 
TABLE I

Corresponding conditions for an arbitrary binary relation $R$ (or " $<$ ") over some set $X$ (of sentences) and its converse complement $X^{2}-R^{-1}$ (or " $\leq$ ")

\begin{tabular}{|c|c|}
\hline $\begin{array}{c}\text { irreflexivity } \\
A \nless A\end{array}$ & $\begin{array}{l}\text { reflexivity } \\
A \leq A\end{array}$ \\
\hline $\begin{array}{c}\text { transitivity } \\
A<B \& B<C \Rightarrow A<C\end{array}$ & $\begin{array}{c}\text { virtual connectivity } \\
A \leq B \Rightarrow A \leq C \text { or } C \leq B\end{array}$ \\
\hline $\begin{array}{c}\text { asymmetry } \\
A<B \Rightarrow B \nless A\end{array}$ & $\begin{array}{c}\text { connectedness } \\
A \leq B \text { or } B \leq A\end{array}$ \\
\hline $\begin{array}{c}\text { continuing up } \\
A<B \& B \vdash C \Rightarrow A<C\end{array}$ & $\begin{array}{c}\text { continuing down } \\
A \leq B \& C \vdash A \Rightarrow C \leq A\end{array}$ \\
\hline $\begin{array}{c}\text { logical truth maximality } \\
\forall A \Rightarrow A<B \text { for some } B\end{array}$ & $\begin{array}{c}\text { logical truth top } \\
B \leq A \text { for every } B \Rightarrow \vdash A\end{array}$ \\
\hline $\begin{array}{l}\text { nonelement minimality } \\
K \neq L \Rightarrow(A \in K \Leftrightarrow B<A \\
\text { for some } B)\end{array}$ & $\begin{array}{c}\text { nonelement bottom } \\
K \neq L \Rightarrow(A \notin K \Leftrightarrow A \leq B \\
\text { for every } B)\end{array}$ \\
\hline $\begin{array}{c}\text { n-acyclicity } \\
A_{1}<A_{2} \& A_{2}<A_{3} \& \ldots \\
\& A_{n-1}<A_{n} \Rightarrow A_{n} \nless A_{1}\end{array}$ & $\begin{array}{c}\text { n-circle } \\
A_{1} \leq A_{2} \text { or } A_{2} \leq A_{3} \text { or } \ldots \\
A_{n-1} \leq A_{n} \text { or } A_{n} \leq A_{1}\end{array}$ \\
\hline $\begin{array}{l}\text { set non-covering } \\
\emptyset \neq H \vdash A \Rightarrow A \nless B \\
\text { for some } B \text { in } H\end{array}$ & $\begin{array}{c}\text { set dominance } \\
\emptyset \neq H \vdash A \Rightarrow B \leq A \\
\text { for some } B \text { in } H\end{array}$ \\
\hline $\begin{array}{l}\text { singleton non-covering } \\
\qquad B \vdash A \Rightarrow A \nless B\end{array}$ & $\begin{array}{c}\text { singleton dominance } \\
B \vdash A \Rightarrow B \leq A\end{array}$ \\
\hline $\begin{array}{c}\text { (non-covering) conjunctiveness } \\
A \wedge B<A \Rightarrow A \wedge B \nless B\end{array}$ & $\begin{array}{l}\text { (dominant) conjunctiveness } \\
A \leq A \wedge B \text { or } B \leq A \wedge B\end{array}$ \\
\hline $\begin{array}{c}\text { conjunctive closure up } \\
A<B \& A<C \Rightarrow A<B \wedge C\end{array}$ & $\begin{array}{c}\text { conjunctive choice down } \\
A \wedge B \leq C \Rightarrow A \leq C \text { or } B \leq C\end{array}$ \\
\hline $\begin{array}{c}\text { entailment closure up } \\
A<B \text { for every } B \text { in } H \neq \emptyset \& \\
H \vdash C \Rightarrow A<C\end{array}$ & $\begin{array}{l}\text { entailment choice down } \\
\emptyset \neq H \vdash B \& B \leq A \Rightarrow \\
C \leq A \text { for some } C \text { in } H\end{array}$ \\
\hline
\end{tabular}


A synopsis of some relevant conditions for relations and their converse complement is given in Table I. Note that the condition of virtual connectivity used in Alchourrón and Makinson (1985) is, on a contrapositive reading, exactly the condition of negative transitivity which plays a prominent role in social choice theory (see Sen 1982). Under some weak additional assumptions, it is equivalent to the conditions of modularity, rankedness, or transitivity of $\sim$ (defined by $A \sim B$ iff neither $A<B$ nor $B<A$ ) which were used by other authors (cf. Lewis 1981, Section 5, Ginsberg 1986, Section 4.4, Lehmann and Magidor 1990, Section 3.6, Makinson 1990, Section 4.1).

The postulates and definition of standard epistemic entrenchment rewritten for the converse complement $<$ of $\leq$

(SEE1) If $A<B$ then $A<C$ or $C<B$

(SEE2) If $B \vdash A$ then $A \nless B$

(SEE3) If $A \wedge B<A$ then $A \wedge B \nless B$

(Virtual Connectivity)

(SEE4) If $K \neq L$ then: $B<A$ for some $B$ iff $A \in K$

(Singleton Non-covering)

(SEE5) If $\forall A$ then $A<B$ for some $B$

(Conjunctiveness)

(Minimality)

(Maximality)

$($ Def $\leq$ ) becomes

(Def $<) A<B$ iff $B \in K \dot{-} A \wedge B$ and $\nvdash A \wedge B$.

(Def-) does not change. In order to reconcile what is intuitively natural (transitivity, but not connectivity) with what is effective in mechanisms for belief revision we shall work with strict relations in the rest of this paper.

\section{BELIEF CHANGE AND EPISTEMIC ENTRENCHMENT: A MORE FLEXIBLE THEORY}

The major changes of the standard theory to be made have already been announced at some length. We are going to get rid of $(\mathrm{K}-4)$ and $(\mathrm{K} \dot{-})$ for contractions, and of (SEE1), (SEE4) and (SEE5) for epistemic entrenchment. We have to investigate what conditions, if any, must appear in their place in order make belief contractions work.

First of all, we shall have to make some minor but decisive changes to every part of the standard theory: to the postulates for contractions and epistemic entrenchment as well as to the bridging principles (Def $<$ ) and (Def- ). To make these minor changes more comprehensible, we note that in the narrower context of the standard theory, they would constitute no changes at all.

Lemma 2 (i) Given $(\mathrm{K} \dot{-})-(\mathrm{K} \dot{-})$, each of the following conditions is equivalent to $(\mathrm{K}-7)$ : 
$(\mathrm{K} \dot{-} 7 \mathrm{r})$ If $K \dot{-} A \neq K$ and $K \dot{-} B \neq K$ then $K \dot{-} A \cap K \dot{-} B \subseteq K \dot{-} A \wedge B$

$(\mathrm{K}-\mathrm{P})$ If $A \in K \dot{-} \wedge \wedge B$ then $A \in K \dot{-A \wedge} B \wedge C$

$(\mathrm{K} \dot{-7 \mathrm{p})}$ If $A \in K \dot{-} A \wedge B \neq K$ then $A \in K \dot{-} A \wedge B \wedge C$

(ii) Given (SEE1), (SEE2) and (SEE3) taken together are equivalent to set non-covering,

(SNC) If $\emptyset \neq H \vdash A$ then $A \nless B$ for some $B$ in $H$

Given (SEE1) and (SEE2), (SNC) is equivalent to entailment closure up,

(ECU) If $A<H \neq \emptyset$ and $H \vdash B$ then $A<B$

(iii) Given $(\mathrm{K}-1)$, $(\mathrm{K}-4)$ and $(\mathrm{Def}<)$, we have $A<B$ iff $B \in K \cdot A \wedge B$ and $A \notin K \dot{-A \wedge B}$.

Moreover, given $(\mathrm{K} \dot{-} 1)-(\mathrm{K} \dot{-8})$ and $(\operatorname{Def}<)$, we have $(B \in K \dot{-} A$ and $A \notin K \dot{-B}) \Rightarrow A<B \Rightarrow B \in K \dot{-} A$.

(iv) Given (SEE1) - (SEE5) and (Def $\dot{-}$ ), we have

$$
K \dot{-} A= \begin{cases}K \cap\{B: A<A \vee B\} & \text { if } A \in K \text { and } A<\top \\ K & \text { otherwise }\end{cases}
$$

" $A<H$ " in the condition (ECU) of course means that $A<C$ for every $C$ in $H$. Essentially $(\mathrm{K}-\mathrm{P})$ is first mentioned as "partial antitony" in Alchourrón, Gärdenfors and Makinson (1985) and the transcription of (SNC) for the converse complement $\leq$ of $<$ is first mentioned in Rott (1991b). In the standard account, then, we have a number of equivalent forms of the definitions used. The equivalences, however, depend on presumptions we would like to give up, viz. on $(\mathrm{K}-4)$ and $(\mathrm{K}-8)$ or on (SEE1), (SEE4) and (SEE5). We find that the standard definitions do not suit our purposes, but we shall be able to use the variants.

Postulates for preferential contractions (over $K$ )

$(\mathrm{K}-1) \quad K \dot{-} A$ is a theory

$(\mathrm{K} \dot{-2}) \quad K \dot{-} A \subseteq K$

$(\mathrm{K}-3) \quad$ If $A \notin K$ then $K \dot{-} A=K$

$(\mathrm{K} \dot{-5}) \quad K \subseteq(K \dot{-} A)+A$

$(\mathrm{K} \dot{-6})$ If $C n(A)=C n(B)$ then $K \dot{-} A=K \dot{-} B$

$(\mathrm{K} \dot{-7 \mathrm{p})}$ If $A \in K \dot{-} A \wedge B$ and $K \dot{-} A \wedge B \neq K$, then $A \in K \dot{-} A \wedge B \wedge C$.

$(\mathrm{K}-7 \mathrm{c})$ If $B \in K \dot{-} \wedge \wedge B$ then $K \dot{-} \subseteq \subseteq K \dot{-} \wedge \wedge B$.

$(\mathrm{K} \dot{\circ} 8 \mathrm{c})$ If $B \in K \dot{-} A \wedge B$ then $K \dot{-} A \wedge B \subseteq K \dot{-}$. 
At least for $(\mathrm{K}-1)$ and $(\mathrm{K}-5)$ it is again essential that they are restricted to the case where $K$ is a theory. The "c" in " $(\mathrm{K}-7 \mathrm{c})$ " and " $(\mathrm{K}-8 \mathrm{c})$ " stands for $\mathrm{cu}$ mulative, or cut and cautious monotony, the " $\mathrm{p}$ " in " $(\mathrm{K}-7 \mathrm{p})$ " for preferential or partial antitony.

A few comments on the motivation of these postulates are in order. First, we removed the success postulate $(\mathrm{K}-4)$ which is related to the maximality postulate (SEE5). We allow a reasoner to refuse the withdrawal of $A$ not only in the case where $A$ is a logical truth. There may well be other sentences ("necessary truths") which are of topmost importance for him. $(\mathrm{K}-2)$ and $(\mathrm{K}-5)$ imply

If $A \in K \dot{-} A$ then $K \dot{-} A=K$.

This is a weak form of $(\mathrm{K}-4)$ and all what we want and need. Notice incidentally that $(\mathrm{K}-1)-(\mathrm{K}-3)$ and $(\mathrm{K}-5)$ imply that

$K \dot{-} A=K$ if and only if $A \notin K$ or $A \in K \dot{-}$.

This fact will be useful for the proof of Theorem 2(iv) below.

Well, what do the new conditions which replace Gärdenfors's supplementary postulates say? The conjunction of $(\mathrm{K}-7 \mathrm{c})$ and $(\mathrm{K}-8 \mathrm{c})$ tells us that if a reasoner who is instructed to withdraw $A \wedge B$ (i.e., to withdraw at least one of $A$ and $B$ ) does not withdraw $B$ then what he does is just withdraw $A$. This seems plausible enough. It is worth mentioning that $(\mathrm{K}-7 \mathrm{c})$ and $(\mathrm{K}-8 \mathrm{c})$ taken together are equivalent to the following reciprocity condition for contractions:

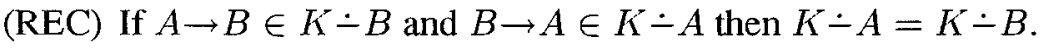

Proof: From $(\mathrm{K} \div 7 \mathrm{c})$ and $(\mathrm{K} \div 8 \mathrm{c})$ to $(\mathrm{REC})$ : Let $A \rightarrow B \in K \div B=$ $K \doteqdot(A \rightarrow B) \wedge(A \vee B)$. Then $(\mathrm{K} \div 7 \mathrm{c})$ and $(\mathrm{K} \div 8 \mathrm{c})$ allow us to conclude $K \dot{-B}=K \dot{-} A \vee B$, and similarly we get $K \dot{-} A=K \dot{-} A \vee B$. From (REC) to $(\mathrm{K} \dot{-} 7 \mathrm{c})$ and $(\mathrm{K} \dot{-} 8 \mathrm{c})$ : Let $B \in K \dot{\circ} \wedge \wedge$. Then, by $(\mathrm{K} \dot{-1})$, $A \rightarrow(A \wedge B) \in K \dot{-} \wedge \wedge$. Moreover, by $(\mathrm{K} \dot{-1}),(A \wedge B) \rightarrow A \in K \dot{-}$.

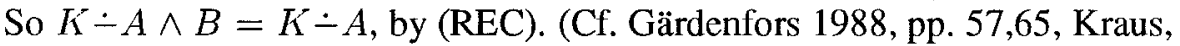
Lehmann and Magidor 1990, p. 179, Makinson and Gärdenfors 1991, p. 198.)

$(\mathrm{K}-7 \mathrm{p})$ tells us that if a reasoner who is instructed to withdraw $A \wedge B$ (i.e., to withdraw at least one of $A$ and $B$ ) does not withdraw $A$ then he or she will not withdraw $A$ when instructed to remove $A \wedge B \wedge C$ (i.e., to withdraw at least one of $A, B$ and $C$ )-provided that he does not simply refuse to withdraw $A \wedge B$. It may happen, however, that the reasoner refuses to withdraw $A \wedge B$ but does not refuse to withdraw $A \wedge B \wedge C$, and after withdrawing $A \wedge B \wedge C A$ is missing in his belief state. $(\mathrm{K}-7 \mathrm{p})$ implies a restricted form of $(\mathrm{K}-7)$, viz.

$(\mathrm{K} \dot{-} 7 \mathrm{r})$ If $K \dot{-} A \neq K$ and $K \dot{-} B \neq K$ then $K \dot{-} A \cap K \dot{-} B \subseteq K \dot{-} A \wedge B$ 
(modify the proof of Observation 3.3 in Alchournón, Gärdenfors and Makinson 1985 accordingly), but in the absence of $(\mathrm{K}-4)$ it does not imply full $(\mathrm{K}-7)$, nor does it imply the weaker $(\mathrm{K} \div 7 \mathrm{c})$. As conversely $(\mathrm{K}-7 \mathrm{c})$ does not entail $(\mathrm{K}-7 \mathrm{p})$, we in fact split up $(\mathrm{K}-7)$ into two weaker versions which do not even jointly imply $(\mathrm{K}-7)$. Unrestricted $(\mathrm{K}-7)$ may fail in our more liberal context for the following reason. It may happen that somebody refuses to withdraw $A$ and refuses to withdraw $B$, but he or she does not refuse to withdraw $A \wedge B$. This does not match well with our idea that withdrawing $A \wedge B$ is the same as withdrawing at least one of $A$ and $B$, but after all in withdrawing $A \wedge B$ the reasoner is at liberty to choose freely between withdrawing $A$ and withdrawing $B$, and that may facilitate the act of withdrawing. Anyway, we will see which role of the various versions of $(\mathrm{K}-7)$ play in the following.

Note that with the exception of $(\mathrm{K}-7 \mathrm{p})$, we are left with Horn conditions only. Had we replaced $(\mathrm{K}-7 \mathrm{p})$ by $(\mathrm{K}-7)$ or $(\mathrm{K}-\mathrm{P})$, we would have the pleasant fact that the intersection of any set of preferential contraction functions is again a preferential contraction function. It is clear that $(\mathrm{K}-8)$ is not derivable from our new set of postulates.

\section{Postulates for generalized epistemic entrenchment}

(GEE1) $T \nless T$

(GEE2 $^{\uparrow}$ ) If $A<B$ and $B \vdash C$ then $A<C$

(GEE2 ${ }^{\downarrow}$ ) If $A<B$ and $C \vdash A$ then $C<B$

(Non-Triviality)

(GEE3 ${ }^{\uparrow}$ ) If $A<B$ and $A<C$ then $A<B \wedge C$

(Continuing Up)

(Continuing Down)

(GEE3 ${ }^{\downarrow}$ ) If $A \wedge B<B$ then $A<B$

(Conjunction Up)

(Conjunction Down)

Let us look at the motivation of (GEE1)-(GEE3 $\left.{ }^{\downarrow}\right)$. (GEE1) is not problematic. (GEE2 ${ }^{\dagger}$ ) says that if $A$ is more exposed to the danger of being withdrawn, or more risky for short, than $B$, and if $B$ implies $C$, then $A$ is more risky than $C$. Loosely speaking, a decrease of logical content always involves a decrease of risk. Similarly, (GEE2 ${ }^{\downarrow}$ ) says that if $A$ is more risky than $B$ and $C$ implies $A$, then $C$ is more risky than $B$. An increase of logical content always involves an increase of risk. Continuing Up and Continuing Down appear in Alchourrón and Makinson $(1985,1986)$, and I take them as minimal conditions of rationality for every relation designed to formalize the concept of epistemic entrenchment.

The conjunctiveness conditions (GEE $3^{\uparrow}$ ) and (GEE $3 \downarrow$ ) which replace the standard conjunctiveness condition (SEE3) rest on the following intuition. As already mentioned, it is both necessary and sufficient to withdraw either $A$ or $B$ (or both) from a belief set $K$ in order to withdraw $A \wedge B$ from $K$. Indeed, it is hardly conceivable what a difference there should be between an 
intention or instruction to withdraw $A \wedge B$ and an intention or instruction to withdraw at least one of $A$ and $B$, with a free choice which of the conjuncts to withdraw. In the following, our guiding hypothesis is that a contraction with respect to a conjunction just is the same as a (finite) choice contraction (this term is due to Fuhrmann 1988). But be cautious. This does not entail that the result of withdrawing $A \wedge B$ is identical with either the result of withdrawing $A$ or the result of withdrawing $B$ or some straightforward combination of these (cf. condition ( $-\mathrm{V})$ of Alchourrón, Gärdenfors and Makinson 1985). We should reckon with the possibility that the simultaneous withdrawal of both $A$ and $B$ is done in a way essentially different from the withdrawal of the single sentences. Now let us turn to the discussion of our postulates. (GEE $3^{\uparrow}$ ) says that if $A$ is more risky than both $B$ and $C$ then it is also more risky than the conjunction $B \wedge C$. If $A$ is given up and $B$ is retained when at least one of $A$ and $B$ must be given up, and if $A$ is given up and $C$ is retained when at least one of $A$ and $C$ must be given up, then $A$ is given up and $B$ and $C$ are retained when at least one of $A, B$ and $C$ must be given up. (GEE3 ${ }^{\downarrow}$ ) says that if $A \wedge B$ is more risky than $B$ then so is $A$ alone. If $A \wedge B$ is given up and $B$ is retained when at least one of $A \wedge B$ and $B$ - i.e., at least one of $A$ and $B !-$ must be given up, then $A$ is given up and $B$ is retained when at least one of $A$ and $B$ must be given up. It will turn out soon that (GEE $3^{\dagger}$ ) and $\left(\mathrm{GEE} 3{ }^{\downarrow}{ }^{\downarrow}\right)$ are very powerful postulates.

Obviously, (GEE $3^{\downarrow}$ ) is only a restricted form of the dual of (GEE3 $\left.3^{\uparrow}\right)$, viz. if $A \wedge B<C$ then $A<C$ or $B<C$.

This condition follows from (SEE1) and (SEE3) in standard epistemic entrenchment. It says that if $A \wedge B-$ i.e., at least one of $A$ and $B$ - is given up and $C$ is retained when at least one of $A \wedge B$ and $C$-i.e., at least one of $A$ and $B$ and $C$-has to be given up, then either $A$ is given up and $C$ is retained when at least one of $A$ and $C$ has to be given up, or $B$ is given up and $C$ is retained when at least one of $B$ and $C$ is to be given up. This seems reasonable, given our interpretation of conjunction contractions as choice contractions. Let us illustrate why we still do not want to accept this condition.

Imagine we are working on the basis of a fictitious miniature medical theory. People are either healthy or ill. Their physical condition is entirely dependent on their being supplied with the right amount of minerals and proteins. There are exactly two indicators of somebody's health, namely blood pressure and pulse. Now normally, in the overwhelming majority of cases, if people are ill, they suffer from a lack rather than from an excess, of either minerals or proteins. Deficiencies are not very dangerous fortunately, but they cause certain symptoms. If you need more minerals, the blood pressure is all right, but the frequency of the pulse is much too low. If you 
need more proteins, the pulse is all right, but the blood pressure is much too high. Sometimes, though, people comsume too much minerals or proteins. As it happens, the symptoms of an excessive consumption of minerals are exactly like those of a deficiency of proteins, and the symptoms of an excessive consumption of proteins are exactly like those of a deficiency of minerals. But beware - excessive consumption is always a serious danger to the patient's life! Now the person we take care of is Henry, and we use the following symbols:

\section{A : Henry's blood pressure is all right. \\ $B$ : Henry's pulse is all right. \\ $C: \quad$ There is no serious danger to Henry's life.}

Our present theory $K$ about Henry says that he is very well, i.e. $K$ contains $A, B$ and $C$. But then Henry feels sick. Something, at least one of $A, B$ and $C$, seems to be wrong. Fortunately we need not worry about Henry too much, because presumably he suffers from a deficiency desease, so he is not very ill, regardless of whether his mineral or protein balance is disturbed. Formally, this should mean that $C \in K \dot{-}(A \wedge B \wedge C)$, or $A \wedge B<C$. But if his blood pressure seems to be very high (while the pulse is still normal), we may be worried. For one possible reason for a high blood pressure is that he has too high a level of minerals, which would indeed mean a serious threat to his life. And similarly, if his pulse seems to be very low (while the blood pressure is still normal), we may again be worried. For one possible reason now is that Henry has too high a level of proteins, which would again be very serious. This kind of informational situation may occur in particular when we do not have any idea about the probability of a disturbance of the minaral metabolism as compared to the probability of a disturbance of the protein metabolism. The ratio of these probabilites might be 1000 to 1 , or 1 to 1000 . Formally, it seems that we are now ready to assent to $C \notin K \dot{-}(A \wedge C)$ and $C \notin K \dot{-}(B \wedge C)$, or $A \nless C$ and $B \nless C$. (Warning: We do not wish to convey the impression that we depend on probability considerations too badly! For instance, (GEE $3^{\dagger}$ ) can only be made sense of in probabilistic terms if we countenance nonstandard numbers as values of probability distributions. It is just that the $A \wedge \neg B \wedge C$ worlds are more plausible that the $\neg A \wedge B \wedge \neg C$-worlds and the $\neg A \wedge B \wedge C$ worlds are more plausible than the $A \wedge \neg B \wedge \neg C$-worlds - and this is all we know.)

In so far as the above line of reasoning is correct, at least as a representation of some cases of application, we had better reject the dual of (GEE $3^{\uparrow}$ ) as a valid pattern of epistemic entrenchment. 
Notice that (GEE1) - (GEE $3^{\downarrow}$ ) leave us with Hom conditions only. As a consequence of this, we have the pleasant fact that the intersection of any set of GEE-relations is again a GEE-relation. This will help us in the discussion of relational belief change in the style of Lindström and Rabinowicz (1991). Another immediate consequence is that the union of any non-empty chain of GEE-relations is again a GEE-relation. This fact will be useful in the proof of Lemma 7 below.

Notice also that in (GEE1) - (GEE $\left.3^{\downarrow}\right)$ no mentioning is made of a specific belief set $K$. Epistemic entrenchment relations are no longer epistemic entrenchment relations with respect to a given theory $K$. Given a GEE-relation, on the other hand, one can obtain different "current theories", according to the requirements of reliability and relevance in a given context. This can be done by choosing a "greatest lower bound" $A$ which just fails to be considered acceptable in that context. Then define $S_{A}=\{B: A<B\} . S_{A}$ is a theory (see $\left(\mathrm{GEE}^{\uparrow}\right)$ in Lemma 3 below), and it is a theory with respect to a given $G E E$ relation $<$. In the standard theory, we invariably have $K=S_{\perp}$. (First steps of considering epistemic entrenchment relations as primary epistemological entities were made in Rott 1991b.)

Since < is transitive (see Lemma $4(v)$ below) $S_{A}$ is an up-set, where an $u p$ set $S$ with respect to some relation < is a collection of objects such that if $A$ is in $S$ and $A<B$ then $B$ is in $S$. Up-sets have a number of nice properties, and they are in fact discussed under different names ("cuts", "fallbacks", "filters") as a means for the direct definition of belief contractions by $K \cdot A=S_{A}$ in Rott (1991a) and Lindström and Rabinowicz (1991). On the other hand, upset contractions notoriously violate the recovery postulate $(\mathrm{K}-5)$ - a fact which may be taken to disqualify them for belief contraction. We shall see, however, that up-sets are well suited for performing belief revision.

For many purposes it will be expedient to make use of a more economic, although less symmetrical, set of postulates.

Lemma 3 The conjunction of $\left(\mathrm{GEE} 2^{\uparrow}\right)$ and $\left(\mathrm{GEE} 3^{\uparrow}\right)$ is equivalent to $\left(\mathrm{GEE}^{\uparrow}\right)$ if $A<H$ and $H \vdash B$ for a non-empty, finite set $H$, then $A<B$

(Closure Up)

The conjunction of $\left(\mathrm{GEE} 2^{\downarrow}\right)$ and $\left(\mathrm{GEE} 3^{\downarrow}\right)$ is equivalent to

$\left(\mathrm{GEE}^{\downarrow}\right)$ if $A<B$ and $C \vdash B \rightarrow A$ then $C<B$

(Closure Down)

GEE-relations are characterizable as relations over $L \times L$ satisfying (GEE1), $\left(\mathrm{GEE}^{\uparrow}\right)$ and $\left(\mathrm{GEE}^{\downarrow}\right)$. Notice that due to the compactness of $C n$, we could equivalently have deleted the restriction of the antecedent of (GEE ${ }^{\uparrow}$ ) to 
finite sets $H$. We choose not to do so, because we will appeal to the finitary character of $\left(\mathrm{GEE}^{\uparrow}\right)$ in the proof of Theorem 3 below.

\section{Lemma 4 (Properties of GEE-relations)}

(i) If $A<B$ and $\vdash A \leftrightarrow A^{\prime}$ and $\vdash B \leftrightarrow B^{\prime}$ then $A^{\prime}<B^{\prime}$

(ii) $A \nless A$

(iii) If $A \wedge C<B \wedge C$ then $A<B$

(iv) If $A<B$ and $C<D$ then $A \wedge C<B \wedge D$

(v) If $A<B$ and $B<C$ then $A<C$

(vi) $A<A \vee B$ iff $A \vee \neg B<A \vee B$

Observe that clauses (ii) and (v) state that $<$ is a strict partial order. It remains to verify that every SEE-relation is a GEE-relation.

Lemma 5 SEE-relations are GEE-relations, or more specifically, (SEE1) - (SEE3) imply (GEE1) - (GEE $\left.3^{\downarrow}\right)$; conversely, (GEE1) - (GEE $\left.{ }^{\downarrow}{ }^{\downarrow}\right)$ imply (SEE2) - (SEE3) and (SNC), but not (SEE1), nor (SEE4) or (SEE5).

Let us consider a simple example that shows the possible failure of virtual connectivity (SEE1) and rational monotony $(\mathrm{K}-8)$. Let $L$ be the propositional language with two atoms $A$ and $B$ and let $C n$ be classical propositional logic. Let the following facts about $<$ be given: $A \vee B<\mathrm{T}, A \vee \neg B<\mathrm{T}$, $\neg A \vee B<T$, and $A<A \vee B$. The most relevant piece of information is the last one. By (GEE ${ }^{\downarrow}$ ), it allows us to deduce that every sentence which entails $A \vee \neg B$ is less entrenched than $A \vee B$. We confine our attention to $<$ restricted to $K=C n(A \wedge B)$ : see Figure 1 . Note that this already exploits the whole power of (GEE1), $\left(\mathrm{GEE}^{\dagger}\right)$ and $\left(\mathrm{GEE}^{\downarrow}\right)$. In particular, nothing allows us to derive either $A<B$ or $B<A \vee B$ from the given information. So the smallest GEE-relation $<$ of which the initial infomation is true does not satisfy virtual connectivity (SEE1). This fact is mirrored

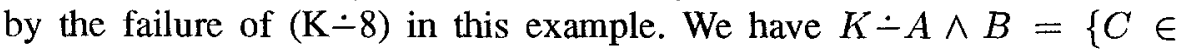

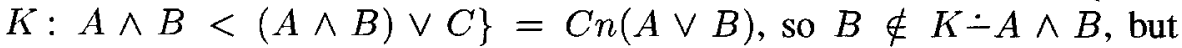

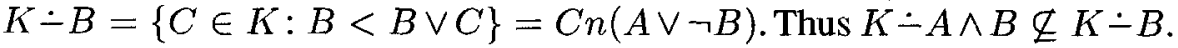

We also consider some supplementary postulates for generalized epistemic entrenchment. Unlike (GEE1) - $\left(\mathrm{GEE} 3^{\downarrow}\right)$, they should not be regarded as constitutive of the concept of epistemic entrenchment.

(GEE4) If $K \neq L$ then: $\perp<A$ iff $A \in K$

(Minimality)

(GEE4') If $A \in K$ and $B \notin K$ then $B<A$

( $K$-Representation)

(GEE5) If $\forall A$ then $A<T$

(Maximality)

(GEE5') If $A<T$ and $B \nless T$ then $A<B$

(Top Equivalence) 


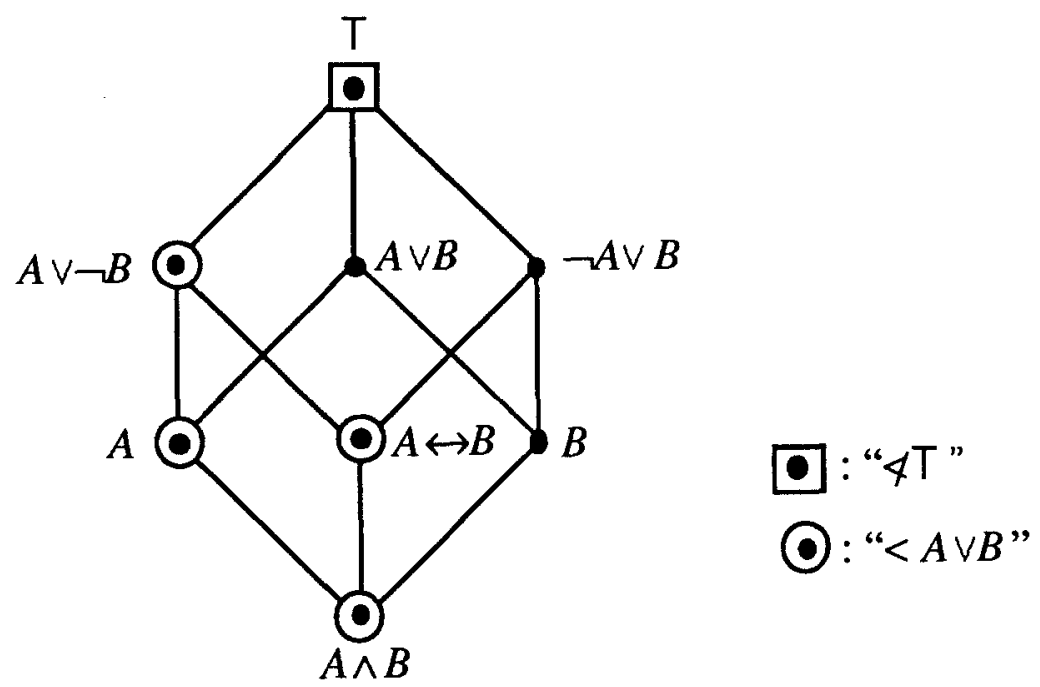

Fig. 1. Example showing the failure of (SEE1) and $(\mathrm{K}-8)$.

Given (GEE2 ${ }^{\uparrow}$ ) and (GEE $2 \downarrow$ ), the minimality and maximality conditions (GEE4) and (GEE5) are obviously equivalent to (SEE4) and (SEE5). (GEE4) and $\left(\mathrm{GEE} 4^{\prime}\right)$ are theory-relative, (GEE5 $\left.{ }^{\prime}\right)$ is non-Hom. (GEE4) and (GEE4') will hold for the redefined $\mathbf{E}(\dot{-})$ and in particular for $\mathbf{E}(\mathbf{C}(<))$. As we shall see later, (GEE5) corresponds to $(\mathrm{K}-4),\left(\mathrm{GEE}^{\prime}\right)$ corresponds to full $(\mathrm{K}-7)$. In the presence of virtual connectivity, (GEE4') follows from (GEE4). Observe that the correspondence between GEE-relations and theories expressed in (GEE4') is many-many: in general, a GEE-relation represents many different theories and a theory is represented by many different GEE-relations. (GEE5') is a special case of virtual connectivity, with $T$ involved in the antecedent. From (GEE1) $-\left(\mathrm{GEE}^{\downarrow}{ }^{\downarrow}\right)$ and $\left(\mathrm{GEE} 5^{\prime}\right)$, it follows that if $A \nless T$ and $B \nless \mathrm{T}$ then $A \wedge B \nless T$. (Proof: Assume that $A \wedge B<T$ and $A \nless T$; then (GEE5') yields $A \wedge B<A$, so $B<A$, by (GEE $3^{\downarrow}$ ), so $B<\mathrm{T}$, by (GEE2 ${ }^{\uparrow}$ ).) It is because (GEE5 $5^{\prime}$ ) is non-Horn and because there is no independent motivation for it in the concept of epistemic entrenchment that we exclude (GEE5') from the set of basic postulates.

It is time to turn to the crucial connection between GEE-relations and contraction operations in belief change. 
From preferential contractions to generalized epistemic entrenchment

(Def $<) \quad A<B$ iff $B \in K \dot{-} \wedge \wedge B$ and $A \notin K \dot{-} A \wedge B$.

From generalized epistemic entrenchment to preferential contractions

(Def-) $K \dot{-} A= \begin{cases}K \cap\{B: A<A \vee B\} & \text { if } A \in K \text { and } A<\top \\ K & \text { otherwise }\end{cases}$

$($ Def $<$ ) is amenable to a "revealed preference" interpretation common in the general theory of choice and preference (see e.g. the "base preferences" in Herzberger 1973). In withdrawing $A \wedge B$ from his current belief set, a reasoner has the obligation to give up at least one of $A$ and $B$, but also the choice between $A$ and $B$. If he chooses to withdraw $A$ while retain $B$ then $A$ is preferred to $B$ under a preference relation of riskiness or vulnerability, or, put differently, $B$ is preferred to $A$ under a preference relation of epistemic entrenchment.

(Def-) would be more straightforward if its crucial phrase were " $A<B$ " instead of " $A<A \vee B$ ". The disjunction is present mainly in order to guarantee satisfaction of the recovery postulate $(\mathrm{K}-5)$. By Lemma 4(vi), $A<A \vee B$ is equivalent to $\neg A \rightarrow \neg B<\neg A \rightarrow B$. Thus we can understand the principal case of (Def-) in the following way. Sentence $B$ is in the contraction of $K$ with respect to $A$ if and only if $B$ is in $K$ and $B$ under the condition $\neg A$ is better entrenched than $\neg B$ under the condition $\neg A$. Roughly, retaining $B$ in $K-A$ means believing that $B$ is true even if it were the case that $\neg A$ is true.

From now on, $\mathbf{E}(\dot{-})$ and $\mathbf{C}(<)$ refer to the epistemic entrenchment relations and contractions defined by the new definitions (Def $<$ ) and (Def - ), respectively. Note that the contraction function defined in (Def - ) is not only dependent on $<$ but also on a belief set $K$. That is, (Def-) specifies the contractions of $K$ for every belief set $K$.

Our main observation reproduces the results of Gärdenfors and Makinson (1988) in the more flexible setting:

Theorem 2 (i) If $<$ satisfies (GEE1) $-\left(\mathrm{GEE} 3^{\downarrow}\right)$ then $\mathbf{C}(<)$ satisfies $(\mathrm{K} \dot{-1})-(\mathrm{K} \dot{-}),(\mathrm{K} \dot{-5}),(\mathrm{K}-6),(\mathrm{K}-7 \mathrm{p}),(\mathrm{K} \dot{-} \mathrm{c})$ and $(\mathrm{K}-8 \mathrm{c})$. If in addition $<$ satisfies (GEE5) then $\mathrm{C}(<)$ satisfies $(\mathrm{K}-4)$; if $<$ satisfies $\left(\mathrm{GEE} 5^{\prime}\right)$ then $\mathrm{C}(<)$ satisfies $(\mathrm{K}-7)$.

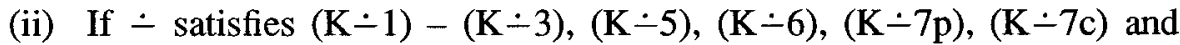
$\left(\mathrm{K} \dot{-8 \mathrm{c})}\right.$ then $\mathrm{E}(\dot{-})$ satisfies $(\mathrm{GEE} 1)-(\mathrm{GEE} 4)$ and $\left(\mathrm{GEE} 4^{\prime}\right)$. If in addition $\dot{-}$ satisfies $(\mathrm{K}-4)$ then $\mathbf{E}(\dot{-})$ satisfies $(\mathrm{GEE} 5)$; if $\dot{-}$ satisfies $(\mathrm{K} \dot{-})$ then $\mathbf{E}(\dot{-})$ satisfies (GEE5'). 
(iii) If $<$ satisfies (GEE1) - (GEE $\left.3^{\downarrow}\right)$ then $\mathbf{E}(\mathbf{C}(<))$ restricted to $K$ is identical with $<$ restricted to $K$. More exactly, with $<^{*}=\mathbf{E}(\mathbf{C}(<))$ :

$$
A<^{*} B \text { iff } B \in K \&(A \notin K \text { or } A<B) \text {. }
$$

If $<$ satisfies (GEE1) - (GEE4) and (GEE4') then $\mathbf{E}(\mathbf{C}(<))=<$.

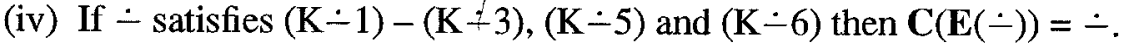

Two comments. First, we see in parts (ii) and (iii) that every GEE-relation $<$ which "comes from" a contraction function over a theory $K$ is also related to $K$, viz. by (GEE4) and (GEE4'). An epistemic entrenchment relation < derived from - over $K$ cannot encode information about sentences outside of $K$, just because - does not care at all about these sentences. Secondly, it is surprising that part (iv) does not depend on any supplementary postulate for contractions. Every contraction function - which satisfies the basic Gärdenfors postulates (except $(\mathrm{K}-4)$ ) admits the (re-)construction of a relation $<=\mathbf{E}(\dot{-})$ which is suitable for application in (Def $\dot{-})$ salva contractione, even if we cannot derive any of the GEE-postulates for < (except (GEE1)).

There is one result of Gärdenfors and Makinson (1988) for SEE-relations which does not hold true for GEE-relations. When a theory $K$ is finite modulo $C n$, every SEE-relation over $K$ is fully determined by its own restriction to the set of coatoms of $K$ (the logically weakest elements of $K-C n(\emptyset)$ ). This is not true for GEE-relations. As a counterexample, consider $K=$ $C n(A \wedge B)$ and a GEE-relation < of which we just know that no element of $\{A \vee B, A \vee \neg B, \neg A \vee B\}$ is better entrenched than any other. This information is compatible with $A<\neg A \vee B$, as well as with $A \nless \neg A \vee B$.

We close this section with a word about revisions. Following the Levi identity, GEE-revisions are defined as $K * A=(K \sqcup \neg A)+A=(K \cap\{B$ : $\neg A<\neg A \vee B\})+A$ for sentences $A$ such that $\neg A \in K$ and $\neg A<\top$, and $K * A=K+A$ otherwise. It is easily verified (cf. Section 5 of Rott 1991a where the proof is carried through for SEE-relations) that this is equivalent to the up-set revision

(Def*) $K * A= \begin{cases}\left(K \cap S_{\neg A}\right)+A & \text { if } \neg A \in K \text { and } \neg A<\top \\ K+A & \text { otherwise }\end{cases}$

(Recall that $S_{\neg A}=\{B: \neg A<B\}$.) Thus GEE-relations provide us with an extremely convenient method of revising beliefs.

GEE-revisions according to (Def*) violate Gärdenfors's consistency preservation postulate saying that $K * A$ must be consistent whenever $A$ is. This corresponds to the violation of the "success postulate" $(K-4)$ for 
contractions. Alternatively, we could think of a reasoner who obeys consistency preservation but may sometimes refuse to revise his belief set. This can be modelled by restricting the Levi identity to cases where the initial contraction is successful (i.e., where $\neg A<\mathrm{T}$ ), and putting $K * A=K$ otherwise.

\section{FUNCTIONAL AND RELATIONAL BELIEF CHANGE}

Belief change in the above sense is functional in that it produces, for every belief set $K$ and every sentence $A$, exactly one revised belief set $K-A$. Lindström and Rabinowicz (1991) have advocated the idea of relational belief revision. If a theory is to be changed in response to new information, they argue, there may be more than just one rational way to do this, without any one of these possibilities being better than any other. This is, of course, a very interesting and legitimate field of examination. But in one respect we are not satisfied if all we know is that, say, $(K \dot{-} A)_{1},(K \dot{-} A)_{2},(K \dot{-} A)_{3}$, etc., are possible ways of removing $A$ from $K$. One answer we would like to get from a model for belief revision is an answer to the question what we should actually do when forced to retract $A$. How should our epistemic state actually look like after retracting $A$ ? A parallel processing of multiple alternative revisions seems to be psychologically unrealistic and computationally impossible, at least if iterated belief changes are taken into account. So the most reasonable advice one can get from the point of view of relational belief revision seems to be the sceptical one: Retain, in the actual contraction $K \dot{-} A$, only those sentences which are left unquestioned in each of the possible ways to perform a rational contraction, i.e., take the intersection $(K \dot{-} A)_{1} \cap(K \dot{-A})_{2} \cap(K \dot{-} A)_{3} \cap \ldots$

Define $B \in K \dot{-} A$ iff $B \in(K \dot{-} A)_{i}$ for every $i$ in some index set $I$. Assuming that each of the candidate contractions is a GEE-contraction, we have in the principal case

$$
\begin{aligned}
& K \dot{-} A= \\
& \bigcap_{i \in I}(K \dot{-} A)_{i}= \\
& K \cap\left\{B: A<_{i} A \vee B \text { for every } i \in I\right\}= \\
& K \cap\{B: A<A \vee B\}, \text { with }<=\bigcap_{i \in I}<_{i} .
\end{aligned}
$$

Since the class of all GEE-relations is closed under intersections, the result of every sceptical relational contraction - based on a collection of GEErelations $<_{i}$ is identical with a simple (functional) contraction based on a GEE-relation, viz. on $\cap\left\{<_{i}: i \in I\right\}$. In particular, every sceptical relational 
contraction based on SEE-relations can be represented as a simple contraction based on a GEE-relation.

It is natural to ask a converse question. If we have a contraction based on some GEE-relation, can it always be represented as the "sceptical" meet of a multitude of SEE-contractions? This reduces to a similar question concerning the underlying relations of epistemic entrenchment. If we have a GEE-relation, can it always be represented as the meet of a suitably chosen set of SEE-relations extending it? Or more ambitiously, is it the case that every GEE-relation is the meet of all its standard extensions?

The only thing that blocks a positive answer is the maximality condition (SEE5). As every standard relation of epistemic entrenchment decides $A<T$ for $A \notin C n(\emptyset)$, so does every meet of SEE-relations. But we wished to drop this requirement for GEE-relations. In order to establish a perfect link, we must either drop (SEE5) for standard relations, or pass from a given GEErelation $<$ to its top extension $<^{\text {top }}=\langle\cup\{\langle A, B\rangle: \forall \forall A$ and $\vdash B\}$ (which is again a GEE-relation). For the sake of simplicity, we take the former option and decide, for the rest of this section, that SEE-relations are not required to satisfy (SEE5). As we will make no reference to a specific theory $K$, we may also largely neglect (SEE4). For every GEE-relation $<\neq \emptyset$, the set $\{A: \perp<A\}$ is a consistent theory, by $\left(\mathrm{GEE}^{\uparrow}\right)$, and it is easy to verify that $<$ satisfies (SEE4) with respect to this set. For the trivial entrenchment relation $<=\emptyset-$ as well as for every other GEE-relation - the trivial theory $K=L$ will do. So we find for every GEE-relation a theory $K$ which is suitable for the fulfilment of (SEE4), and this is all we require from the present perspective. The main object of interest in this section, then, is virtual connectivity (SEE1).

In order to achieve our task, we first address ourselves to a question which is interesting in itself. Suppose you have an arbitrary "base relation" $R \subseteq L \times L$ consisting of pairs of sentences $\langle A, B\rangle$ such that, intuitively speaking, $A$ is epistemically less firmly entrenched than $B$. How can we know that this base relation can serve as the foundation of a full-fledged, formal relation of epistemic entrenchment, in either the standard or the generalized sense? What we have to do is investigate whether $R$ can be extended to a SEE- or GEE-relation. The following theorem tells us how to check this.

Theorem 3 Let $R$ be a binary relation over $L$. Then $R$ can be extended to a GEE-relation iff it satisfies the following condition of entrenchment consistency:

(EC) There is no finite set $\left\{\left\langle A_{1}, B_{1}\right\rangle, \ldots,\left\langle A_{n}, B_{n}\right\rangle\right\} \subseteq R$ such that $B_{1} \wedge \ldots \wedge B_{n} \vdash A_{1} \wedge \ldots \wedge A_{n}$.

(Cf. Rott 1991b, Observation 3 and Definition 12.) Let us say that a binary relation $R$ over $L$ is entrenchment consistent, or simply consistent, if it can 
be extended to a GEE-relation over $L$. For any GEE-relation $<$, we say that a pair $\langle A, B\rangle$ is consistent with $<$ iff $\langle\cup\{\langle A, B\rangle\}$ is consistent, otherwise we say that $\langle A, B\rangle$ is inconsistent with $<$. We then have the following useful

Lemma 6 A pair $\langle A, B\rangle$ is inconsistent with a GEE-relation $<$ iff $B<$ $B \rightarrow A$.

For the next lemma, we use the fact that $L$ is supposed to be a countable language.

Lemma 7 Every GEE-relation can be extended to a SEE-relation. An immediate corollary of Theorem 3 and Lemma 7 is

Lemma 8 Let $R$ be a binary relation over $L$. Then $R$ can be extended to a SEE-relation iff it satisfies the condition of entrenchment consistency.

At last it is no longer difficult to show that our aim can by achieved.

Theorem 4 Every GEE-relation is the meet of all SEE-relations (which are not required to satisfy (SEE5)) extending it.

This result is similar to Lindström and Rabinowicz's (1991) Theorem 3.14. Due to different formalizations of epistemic entrenchment, however, our proof is entirely different from the proof given there.

\section{BELIEF CONTRACTION MODELS}

The Alchourrón-Gärdenfors-Makinson approach considers contraction functions over a fixed belief set $K$. Accordingly, the standard theory of epistemic entrenchment studies relations which are likewise related to some fixed belief set $K$-witness condition (SEE4). Our theory relieves us of this dependency. We can use one and the same GEE-relation $<$ for treating contractions $\mathbf{C}(<, K)$ of various belief sets $K$ rather than just one $\mathbf{C}(<)$ for a fixed $K$. In particular, it is possible to model iterated changes of belief. The information encoded in a GEE-relation exceeds the information encoded in a contraction function over some fixed consistent $K$. It is rather equivalent to a family of contraction functions, one contraction function for every belief set $K$, or, to put it differently, to a belief contraction model. Here a belief contraction model is meant to be a pair $\langle\mathcal{K},-\dot{-}\rangle$, where $\mathcal{K}$ is a non-empty set of belief sets and $-: \mathcal{K} \times L \rightarrow \mathcal{K}$ is a function assigning to every belief set $K$ in $\mathcal{K}$ and every sentence $A$ a unique contraction $K \dot{-} A$ in $\mathcal{K}$. (Belief revision models are discussed by Gärdenfors and others in the context of Ramsey test inter- 
pretations of conditionals. Also cf. Alchourrón and Makinson 1985, Section 7.)

It is worthwhile to take down some of the most basic properties of the belief contraction models generated by GEE-relations. Obviously, we have

$$
K \dot{-} A= \begin{cases}K \cap(L-A) & \text { if } A \in K \text { and } A<\top \\ K & \text { otherwise }\end{cases}
$$

So after all it turns out that all we need to know for the contraction of an arbitrary belief set $K$ is how to contract the "absurd theory" $L$. Belief contraction models reduce to single contraction functions over $L$. Note that the recovery postulate $(\mathrm{K}-5)$ for $L$ means that $\neg A$ has to be in $L \dot{-A}$. For the absurd theory $L$, contraction with respect to $A$ coincides with revision by $\neg A$. The other postulates for preferential contractions do not seem to have a particularly interesting special impact on the contraction of $L .(\mathrm{K}-2)$ and $(\mathrm{K}-3)$ are trivial for $K=L$.

The standard equation $K=S_{\perp}$ receives a new interpretation from this perspective. For any non-empty GEE-relation $<, S_{\perp}=L-\perp$. Hence on the standard account, $K=L \dot{-}$. This means that the standard "current theory" $K$ entertained by an epistemic subject may be regarded as the result of removing inconsistency from the multiplicity of informations he or she has received - and in practice this multiplicity is very likely to be inconsistent.

We have a qualified monotony principle for contractions: If $K \subseteq K^{\prime}$ and $A \in K$ then $K \dot{-} A \subseteq K^{\prime} \dot{-}$. Although this seems to be quite reasonable, it has to be checked against intuitive examples. Gärdenfors (1988, pp. 59-60 and 65) argues against an unqualified principle of monotony (viz., if $K \subseteq K^{\prime}$ then $K \dot{-} \subseteq K^{\prime}-A$ ), and the counterexample he puts forward is in fact a case where $\bar{A} \notin K$. For a discussion of the significance of this unqualified principle, see Katsuno and Mendelson (1992) and Morreau and Rott (1991).

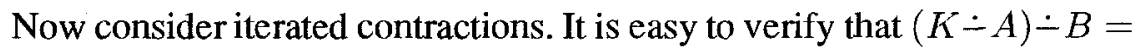
$K \dot{-} \cap \cap K \dot{-} B$, unless $B \notin K \dot{-}$, or more precisely, unless $A \in K, A<\top$ and $A \nless A \vee B$. In the latter case, $(K \dot{-A}) \dot{-B}=K \dot{-}$. It is this case (and its analogue for $(K \dot{-} B) \dot{-}$ ) which blocks the satisfaction of the order independence condition $(K \dot{-} A)-B=(K \dot{-} B)-A$.

\section{CONCLUSION}

This paper has made a step towards the justification of the claim that with epistemic entrenchment one can do anything which can be done by other established methods of belief change - even if one does not accept the entire set of the Gärdenfors postulates. We have removed three unwelcome restrictions, viz. virtual connectivity, minimality and maximality, from the theory of 
epistemic entrenchment as developed by Gärdenfors and Makinson. Our generalized relation conforms to the basic idea of epistemic entrenchment spelled out in the introduction (cf. (Def- )), and it permits the same kind of simple construction of belief contractions and revisions as the more demanding relation of Gärdenfors and Makinson (cf. (Def $<$ ) and (Def $*)$ ).

Besides epistemic entrenchment contractions, a number of other methods for constructing contractions have been propounded in the literature. It is an interesting task to check which of the postulates for preferential contractions are satisfied by which of these constructions. In particular it is natural to ask about the systematic value of the rather new postulate $(\mathrm{K}-8 \mathrm{c})$. Another idea is to extract directly a relation of epistemic entrenchment from the informational basis of these alternative methods. For safe contractions in the sense of Alchourrón and Makinson $(1985,1986)$, relevant work has been done in Rott (1992). For partial meet contractions without transitivity of the underlying non-strict relations (see Alchourrón, Gärdenfors and Makinson 1985), it seems promising to generalize the account of Rott (1991a). For full meet contractions of theory bases (see Veltman 1976, Kratzer 1981, Lewis 1981), it seems clear that epistemic entrenchment reconstructions are possible only if satisfaction of the recovery postulate $(\mathrm{K}-5)$ is guaranteed in a somewhat artificial way (cf. Nebel 1989). Still another undertaking is the invention of a semantics for belief contraction. More specifically, one may look for a semantics in the style of Kraus, Lehmann and Magidor (1990), which bears an intimate relation to the theory of partial meet contractions of Alchourrón, Gärdenfors and Makinson. However, all of these projects require too much care and elaboration to be addressed here even in outline.

\section{ACKNOWLEDGEMENT}

I should like to thank an anonymous referee and David Makinson for many very valuable comments on an earlier version of this paper.

\section{APPENDIX: PROOFS}

Proof of Lemma 1. Assume that $(\mathrm{K}-1)-(\mathrm{K}-6)$ and the Levi identity hold. It is proven in Gärdenfors (1988), Theorem 3.3, that $(\mathrm{K}-7)$ is equivalent to (Dis) and $(\mathrm{K}-8)$ is equivalent to $(\mathrm{RM})$. We prove the rest.

$(\mathrm{Cut})$ is equivalent to $(\mathrm{K} \dot{-} \mathrm{C})$ : Using the Levi identity, we see that (Cut) says

If $B \in(K \dot{\succ} \neg A)+A$ and $C \in(K \dot{\neg} \neg A \vee \neg B)+A \wedge B$ then $C \in$ $(K \dot{-} \neg A)+A$. 
Switching the negations of $A$ and $B$ and applying the deduction theorem for $C n$, we get that this is

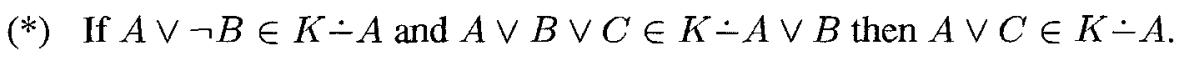

To show that $(*)$ implies $(\mathrm{K}-7 \mathrm{c})$, assume that $D \in K \dot{-} D \wedge E$ and $F \in K \dot{-}$. We need to show that $F \in K \dot{-} \wedge \wedge E$. By $(\mathrm{K} \dot{-1})$, we have $(D \wedge E) \vee(D \vee \neg E) \in K \dot{\circ} \wedge E$. So, by $(*)$, if $(D \wedge E) \vee \neg(D \vee$ $\neg E) \vee C \in K \dot{-}(D \wedge E) \vee \neg(D \vee \neg E)$ then $(D \wedge E) \vee C \in K \dot{-} D \wedge E$. Since $(D \wedge E) \vee \neg(D \vee \neg E)$ is logically equivalent with $E$, we have that if $E \vee C \in K \dot{-}$ then $(D \wedge E) \vee C \in K \dot{-} D \wedge E$. Now by $F \in K \dot{-} E$ and $(\mathrm{K}-1), E \vee F \in K \dot{-}$. So $(D \wedge E) \vee F \in K \dot{-} \wedge \wedge$. But by $(\mathrm{K}-2)$,

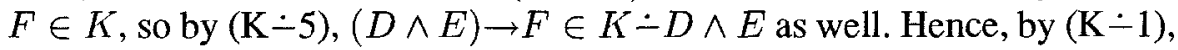
$F \in K \sqcup D \wedge E$.

To show that conversely $(\mathrm{K}-7 \mathrm{c})$ implies $(*)$, assume that $A \vee \neg B \in K \dot{-} A$

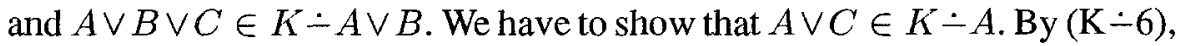

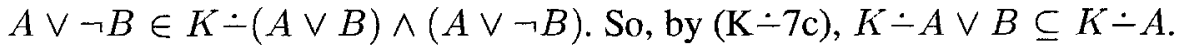
Thus $A \vee B \vee C \in K \dot{-}$. But also $A \vee \neg B \in K \dot{-}$. Thus, by (K-1), $A \vee C \in K \dot{-} A$.

$(C M)$ is equivalent to $(K \dot{-} 8 \mathrm{c})$ : Applying the Levi identity, switching the negations of $A$ and $B$ and using the deduction theorem for $C n$, we see that (CM) says

(**) If $A \vee \neg B \in K \dot{-} A$ and $A \vee C \in K \dot{-} A$ then $A \vee B \vee C \in K \dot{-} A \vee B$.

To show that $(* *)$ implies $(\mathrm{K}-8 \mathrm{c})$, assume that $D \in K \dot{-} D \wedge E$ and $F \in K \dot{-} D \wedge E$. We need to show that $F \in K \dot{-}$. Proceding as in the case

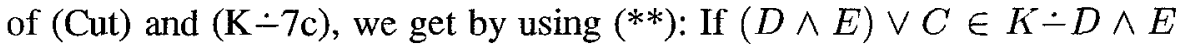

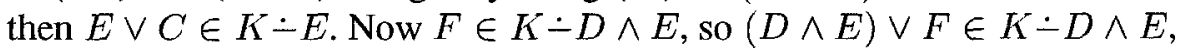

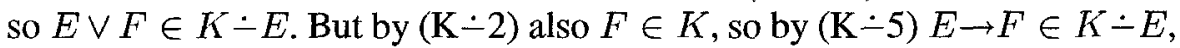
so, by $(\mathrm{K} \dot{-1})$ again, $F \in K \dot{-}$.

To show that conversely $(\mathrm{K}-8 \mathrm{c})$ implies (**), assume that $A \vee \neg B \in$

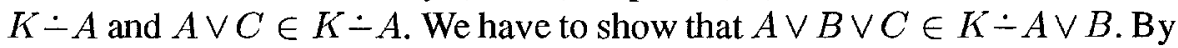
$(\mathrm{K} \dot{-} 6), A \vee \neg B \in K \dot{-}(A \vee B) \wedge(A \vee \neg B)$. So by $(\mathrm{K} \dot{-} 8 \mathrm{c}), K \dot{-} A \subseteq K \dot{-} A \vee B$.

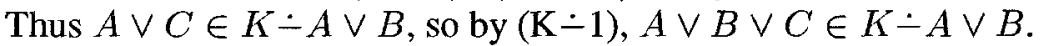

We also give direct proofs of $(\mathrm{K} \div 7) \Rightarrow(\mathrm{K}-7 \mathrm{c})$ and $(\mathrm{K}-8) \Rightarrow(\mathrm{K} \dot{-} 8 \mathrm{c})$.

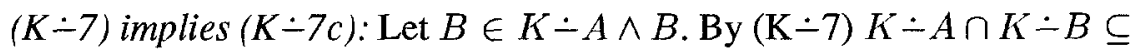
$K \dot{-} A \wedge B$, hence $(K \dot{-} A)+B \cap(K \dot{-} B)+B \subseteq(K \dot{\circ} \wedge \wedge B)+B$. But

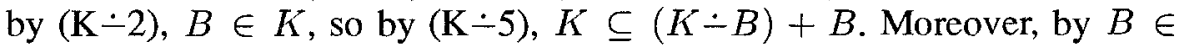
$K \dot{-} A \wedge B,(K \dot{-} A \wedge B)+B=K \dot{-} A \wedge B$, so $K \dot{-} A=K \dot{-} \cap \cap \subseteq$ $(K \dot{-} A)+B \cap(K \dot{-} B)+B \subseteq K \dot{-} A \wedge B$. 
$(K \dot{\circ} 8)$ implies $(K \dot{-} 8 c)$ : Let $B \in K \dot{-} \wedge \wedge B$. By $(\mathrm{K} \dot{-} 4)$, either $A \wedge B \notin$ $K \dot{-} \wedge \wedge B$ or $\vdash A \wedge B$. In the former case, $A \notin K \dot{-} \wedge \wedge B$, by (K-1), so we

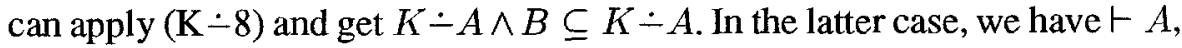

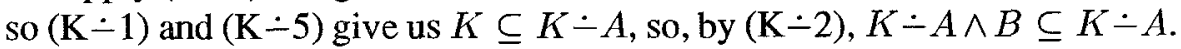
(Note that this proof depends on $(\mathrm{K}-4)$.)

Proof of Lemma 2. (i) Let $(\mathrm{K} \dot{-1})-(\mathrm{K} \dot{-} 6)$ be given.

$(K \dot{-7})$ is equivalent to $(K \dot{-} 7 r)$ : That $(\mathrm{K}-7)$ implies the restricted form $(\mathrm{K}-7 \mathrm{r})$ is obvious. To show that $(\mathrm{K}-7 \mathrm{r})$ implies $(\mathrm{K}-7)$, suppose that $K-A=$ $K$. Then, by (K-4), either $A \notin K$ or $\vdash A$. In the first case $A \wedge B \notin K$, so $(\mathrm{K}-3)$ gives us $K \dot{-} A \wedge B=K$, so $(\mathrm{K} \div 7)$ follows from $(\mathrm{K}-2)$. In the

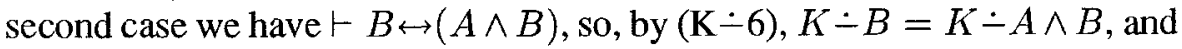
$(\mathrm{K} \div 7)$ follows trivially. The case $K-B=K$ is similar.

$(K \dot{-})$ is equivalent to $(K \dot{-P}):(\mathrm{K} \dot{-P})$ is virtually the same as the "partial antitony" condition

$(\dot{-P})(K \dot{-} A) \cap C n(A) \subseteq K \dot{-} A \wedge B$

of Alchourrón, Gärdenfors and Makinson (1985). That $(\dot{-P})$ implies $(\mathrm{K}-\mathrm{P})$ is immediate. To see that $(\mathrm{K} \dot{-} \mathrm{P})$ implies $(\dot{-P})$, let $A \vdash C$ and $C \in K \dot{-}$. Thus,

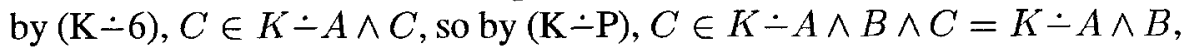
by $(\mathrm{K}-6)$ again. So the claim is identical with the claim of Observation 3.3 of Alchourrón et al., and we refer to the proof given there. Recovery $(\mathrm{K}-5)$ enters essentially into that proof.

$(K \dot{-P})$ is equivalent to $(K-7 p)$ : That $(\mathrm{K}-\mathrm{P})$ implies the restricted form $(\mathrm{K}-7 \mathrm{p})$ is obvious. To show that $(\mathrm{K}-7 \mathrm{p})$ implies $(\mathrm{K}-\mathrm{P})$, suppose that $A \in$

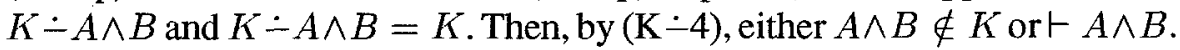
In the first case, $A \wedge B \wedge C \notin K$, so $A \in K \dot{-} \wedge \wedge \wedge C$ follows from (K-2) and $(\mathrm{K}-3)$. In the second case, we have $\vdash A$ and $A \in K \dot{-} \wedge \wedge \wedge C$ follows from $(\mathrm{K}-1)$.

(ii) Let (SEE1) be given.

(SEE2)\&(SEE3) is equivalent to (SNC): That (SNC) implies (SEE2) and (SEE3) is immediate if we put $H=\{B\}$ and $H=\{A, B\}$ respectively.

It remains to show that (SEE1) - (SEE3) imply (SNC). Let $\emptyset \neq H \vdash A$. So, by the compactness of $C n$, there are $B_{1}, \ldots, B_{n} \in H$ such that $B_{1} \wedge \ldots \wedge B_{n} \vdash$ $A$. So by (SEE2), $A \nless B_{1} \wedge \ldots \wedge B_{n}$. Now suppose for reductio that $A<B_{i}$ for every $i=1, \ldots, n$. By (SEE3), we have $B_{1} \wedge B_{2} \nless B_{1}$ or $B_{1} \wedge B_{2} \nless B_{2}$. So by $A<B_{1}$ and $A<B_{2}$ and (SEE1), $A<B_{1} \wedge B_{2}$. By (SEE3), we have $B_{1} \wedge B_{2} \wedge B_{3} \nless B_{1} \wedge B_{2}$ or $B_{1} \wedge B_{2} \wedge B_{3} \nless B_{3}$. So by $A<B_{1} \wedge B_{2}$ and $A<B_{3}$ and (SEE1), $A<B_{1} \wedge B_{2} \wedge B_{3}$. By repetition of this reasoning, we get $A<B_{1} \wedge \ldots \wedge B_{n}$, and we have a contradiction.

Now let (SEE1) and (SEE2) be given. 
(SNC) is equivalent with (ECU): To see that (SNC) implies (ECU), let $A<H \neq \emptyset$ and $H \vdash B$. We need to show that $A<B$. But (SNC) gives us that $B \nless \mathrm{C}$ for some $C$ in $H$. Thus, by $A<C$ and (SEE1), $A<B$.

To see that (ECU) implies (SNC), let $\emptyset \neq H \vdash A$. We need to show that $A \nless B$ for some $B$ in $H$. Suppose for reductio that $A<B$ for every $B$ in $H$. Then (ECU) gives us $A<A$, contradicting (SEE2).

(iii) By (Def $<$ ), we have to show that $B \in K \dot{\triangle} \wedge B \& \nvdash \forall \wedge B$ is

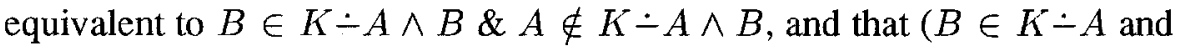
$A \notin K \dot{-B}) \Rightarrow(B \in K \dot{-} A \wedge B$ and $A \notin K \dot{-} A \wedge B) \Rightarrow B \in K \dot{-A}$. Let $(\mathrm{K} \dot{-1})$ and $(\mathrm{K} \dot{-4})$ be given.

$B \in K \dot{-A} \wedge B \& \forall A \wedge B$ is equivalent to $B \in K-A \wedge B \& A \notin$ $K \dot{-} A \wedge B$ :

To see that the former implies the latter, assume that $B \in K \dot{-} \wedge B$ and $A \in K \dot{-} \wedge \wedge B$. Then, by $(\mathrm{K}-1), A \wedge B \in K \dot{-} \wedge \wedge B$, so by $(\mathrm{K}-4), \vdash A \wedge B$.

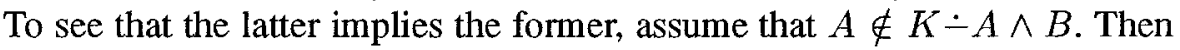
by $(\mathrm{K}-1) \not A$, so $\nvdash A \wedge B$.

Now let $(\mathrm{K} \dot{-1})-(\mathrm{K} \dot{-})$ be given.

$(B \in K \dot{-} A$ and $A \notin K \dot{-} B) \Rightarrow(B \in K \dot{-} A \wedge B$ and $A \notin K \dot{-} A \wedge B) \Rightarrow$ $B \in K \dot{-} A$ :

The limiting case $A \notin K$ is easy. So assume that $A \in K$. Let $B \in K \sqcup A$

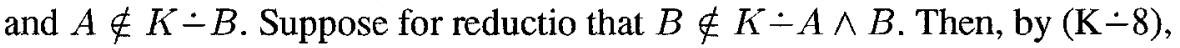

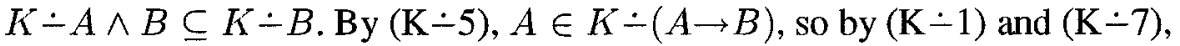
$A \vee B \in K \dot{-} A \cap K \dot{-}(A \rightarrow B) \subseteq K \dot{-} A \wedge B$. So $A \vee B \in K \dot{-}$. But also, by $(\mathrm{K} \dot{-5}), B \rightarrow A \in K \dot{-} B$. Thus, by $(\mathrm{K} \dot{-}), A \in K \dot{-} B$, and we have a contradiction. So we have $B \in K \dot{ } A \wedge B$. Next suppose for reductio that also $A \in K \dot{\circ} \wedge \wedge$. But then $A \wedge B \in K \dot{\circ} \wedge B$, so (K-4) gives us

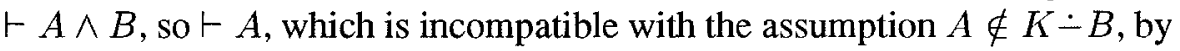
$(\mathrm{K}-1)$.

Now let $B \in K \sqcup A \wedge B$ and $A \notin K \sqcup A \wedge B$. The last condition and $(\mathrm{K}-8)$ give us $K \doteq A \wedge B \subseteq K \sqcup A$, so by the first condition $B \in K \dot{-}$. (We do not, however, get $A \notin K \dot{-B}$.)

(iv) If $\vdash A$ then $A \nless \mathrm{T}$, by (SEE2), and $K \dot{-} A=K$, by (Def-). If $\forall A A$ then $K \dot{-} A=K \cap\{B: A<A \vee B\}$, by (Def- ). We are done if $A \in K$ and $A<\mathrm{T}$. So assume that $A \notin K$, and let $B \in K$. Since $K$ is a theory, also $A \vee B \in K$. Then by (SEE4) $C<A \vee B$ for some $C$. But by (SEE4) again, $C \nless A$, hence, by (SEE1) $A<A \vee B$. So $K \subseteq\{B: A<A \vee B\}$, i.e., $K=K \cap\{B: A<A \vee B\}$ which is what we need. Now assume that $A \nless \top$. For every sentence $B$, we have $B \vdash \top$, so by (SEE2) $\top \nless B$. Taking $A \nless \top$ and $\top \nless B$, (SEE1) gives us $A \nless B$, for arbitrary $B$. Thus, (SEE5) gives us $\vdash A$, and that case we have already treated.

Proof of Lemma 3. It is trivial that $\left(\mathrm{GEE}^{\dagger}\right)$ implies $\left(\mathrm{GEE} 2^{\uparrow}\right)$ and $\left(\mathrm{GEE} 3^{\uparrow}\right)$. 
To show conversely that (GEE $2^{\uparrow}$ ) and (GEE $3^{\dagger}$ ) jointly imply (GEE ${ }^{\uparrow}$ ), let $H$ be a non-empty, finite set of sentences such that $A<H$ and $H \vdash B$. The latter says that there are $C_{1}, \ldots, C_{n} \in H$ such that $C_{1} \wedge \ldots \wedge C_{n} \vdash B$. By repeated application of $($ GEE $3 \uparrow)$, we get $A<C_{1} \wedge \ldots \wedge C_{n}$, and by (GEE2 $2^{\uparrow}$ ), we get $A<B$. as desired. - It is trivial that (GEE ${ }^{\downarrow}$ ) implies (GEE2 ${ }^{\downarrow}$ ). To see that it also implies (GEE ${ }^{\downarrow} \downarrow$ ), let $A \wedge B<B$. Since $A \vdash B \rightarrow(A \wedge B$ ), (GEE ${ }^{\downarrow}$ ) gives us $A<B$. To show conversely that (GEE2 $\downarrow$ ) and (GEE3 ${ }^{\downarrow}$ ) jointly imply (GEE $\left.{ }^{\downarrow}\right)$, let $A<B$ and $C \vdash B \rightarrow A$. Since $(B \rightarrow A) \wedge B \vdash A$, (GEE2 ${ }^{\downarrow}$ ) gives us $(B \rightarrow A) \wedge B<B$, from which we get, using (GEE3 ${ }^{\downarrow}$ ), that $B \rightarrow A<B$. But then, since $C \vdash B \rightarrow A$, (GEE2 ${ }^{\downarrow}$ ) finally yields $C<A$, as desired.

Proof of Lemma 4. (i) Immediate from (GEE2 ${ }^{\uparrow}$ ) and (GEE2 ${ }^{\downarrow}$ ).

(ii) Suppose for reductio that $A<A$. Then by (GEE2 $\downarrow$ ), $\mathrm{T} \wedge A<A$, so by (GEE $3^{\downarrow}$ ) $T<A$, hence by (GEE2 $\left.{ }^{\uparrow}\right) \top<T$, contradicting (GEE1).

(iii) Let $A \wedge C<B \wedge C$. By (GEE2 $\left.{ }^{\downarrow}\right), A \wedge B \wedge C<B \wedge C$, so by (GEE3 $\left.{ }^{\downarrow}\right)$ $A<B \wedge C$, so by $(\mathrm{GEE} 2 \uparrow) A<B$.

(iv) Let $A<B$ and $C<D$. By (GEE2 $\left.{ }^{\downarrow}\right), A \wedge C<B$ and $A \wedge C<D$. Hence, by $\left(\mathrm{GEE} 3^{\uparrow}\right), A \wedge C<B \wedge D$.

(v) Let $A<B$ and $B<C$. Then, by (iv) and (GEE2 $\left.{ }^{\uparrow}\right), A \wedge B<C \wedge B$. So $A<C$, by (iii).

(vi) From left to right: Let $A<A \vee B$. Then by (GEE $\downarrow)(A \vee B) \rightarrow A<$ $A \vee B$, so by (i) $A \vee \neg B<A \vee B$. From right to left: By (GEE2 ${ }^{\downarrow}$ ).

Proof of Lemma 5. SEE-relations are GEE-relations: (GEE1) is implied by (SEE2). That (GEE ${ }^{\uparrow}$ ) is implied by (SEE1) - (SEE3), is stated in Lemma 2(ii). For (GEE2 $\downarrow$ ), assume that $A<B$ and $C \vdash A$. From the latter, we conclude with (SEE2) that $A \nless C$, so by the former and (SEE1) $C<B$, as desired. For (GEE3 ${ }^{\downarrow}$ ), assume that $A \wedge B<B$. Then, by (SEE3), $A \wedge B \nless A$. Hence, by (SEE1), $A<B$, as desired.

(GEE1)-(GEE3 $\left.{ }^{\downarrow}\right)$ imply (SEE2) - (SEE3) and (SNC) but neither (SEE1) nor (SEE4) nor (SEE5). For (SEE2), suppose for reductio $B \vdash A$ and $A<B$. Then by (GEE2 ${ }^{\uparrow}$ ) $A<A$, contradicting Lemma 4(ii). For (SEE3), suppose for reductio that $A \wedge B<A$ and $A \wedge B<B$. By (GEE3 ${ }^{\uparrow}$ ), we get $A \wedge B<A \wedge B$, contradicting Lemma 4(ii). For (SNC), suppose for reductio that $A<H$. Then, by $H \vdash A$ and (GEE ${ }^{\dagger}$ ), $A<A$, contradiction Lemma 4(ii). That GEErelations do not generally satisfy (SEE4) and (SEE5) is immediate from the fact that $<=\emptyset$ is a GEE-relation. A counterexample to (SEE1) is given in the text just after Lemma 5.

Proof of Theorem 2. (i) Let $K$ be a theory and < satisfy (GEE1) $\left(\mathrm{GEE}^{\downarrow}{ }^{\downarrow}\right)$.

$(\mathrm{K}-1)$ : It is trivial that $K \dot{-} A$ is a theory if $A \notin K$ or $A \nless T$. Otherwise, $K \dot{-} A=K \cap\{B: A<A \vee B\}$. As $K$ is a theory, it suffices to show that 
$\{B: A<A \vee B\}$ is a theory. Let $A<A \vee B_{i}$ for every $i$ in some index set $I$ and $\left\{B_{i}: i \in I\right\} \vdash B$. Then, by compactness, $\left\{B_{i}: i \in I_{0}\right\} \vdash B$ for some finite $I_{0} \subseteq I$, and $\left\{A \vee B_{i}: i \in I_{0}\right\} \vdash A \vee B$, so $A<A \vee B$ by $\left(\mathrm{GEE}^{\uparrow}\right)$, and we are done.

$(\mathrm{K} \dot{-2})$ and $(\mathrm{K} \dot{-})$ are obvious from (Def-).

(K-5): If $A \notin K$ or $A \nless \mathrm{T}$, this is obvious. So assume that $A \in K$, $A<T$ and $C \in K$. Since $C n$ satisfies the deduction theorem, we have to show that $A \rightarrow C \in K \dot{-}$, i.e., by (Def-), $A \rightarrow C \in K$ and $A<A \vee(A \rightarrow C)$. But the former follows from the fact that $C \in K$ and $K$ is a theory, and the latter follows from $A<\mathrm{T}, T \vdash A \vee(A \rightarrow C)$ and (GEE2 $\uparrow$ ).

$(\mathrm{K}-6)$ follows from Lemma 4(i).

$(\mathrm{K}-7 \mathrm{p})$ : Let $A \in K \dot{-} \wedge B \neq K$. By (Def-) then, $A \wedge B \in K$ and $A \wedge B<T$ and $A \wedge B<(A \wedge B) \vee A$, i.e., by (GEE2 $\left.{ }^{\uparrow}\right), A \wedge B<A$. If $K \dot{-} \wedge \wedge \wedge C=K$, then $A \in K \dot{-} \wedge \wedge \wedge C$, because $A \wedge B \in K$. So assume that $K \dot{-} \wedge \wedge \wedge C \neq K$. Then $A \wedge B \wedge C \in K$ and $A \wedge B \wedge C<T$. We have to show that $A \wedge B \wedge C<(A \wedge B \wedge C) \vee A$, i.e., $A \wedge B \wedge C<A$. But this follows from $A \wedge B<A$ and (GEE2 $\downarrow$ ).

$(\mathrm{K}-7 \mathrm{c})$ : Let $B \in K \dot{-} A \wedge B$ and $C \in K \dot{-}$. We need to show that $C \in K \dot{-} A \wedge B$. If $K \dot{-} A \wedge B=K$, then we are done, by $C \in K \dot{-} A$ and (Def- $)$. So let $K \dot{-} A \wedge B \neq K$. Then $A \wedge B \in K$ and $A \wedge B<T$. Now $B \in K \dot{-A} \wedge B$ says that $A \wedge B<B$, so, by (GEE $3^{\downarrow}$ ), $A<B$, so, by $\left(\mathrm{GEE} 2^{\uparrow}\right.$ ), $A<\mathrm{T}$. As also $A \in K, C \in K \dot{-}$ s says that $A<A \vee C$. By (GEE2 $\downarrow$ ), then, $A \wedge B<A \vee C$. Together with $A \wedge B<B$, this gives us $A \wedge B<(A \vee C) \wedge B$, by $\left(\mathrm{GEE} 3^{\uparrow}\right)$, so by $\left(\mathrm{GEE} 2{ }^{\uparrow}\right)$ again, $A \wedge B<(A \wedge B) \vee C$. But this means that $C \in K \dot{-A} \wedge B$, as desired.

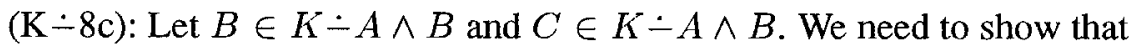
$C \in K \dot{-}$. If $K \dot{-} A=K$ then we are done, by $C \in K \dot{-} A \wedge B$ and (Def $\dot{-}$ ). So let $K \dot{-} A \neq K$. Then $A \in K$ and $A<\mathrm{T}$. Since $B \in K \dot{-} \wedge \wedge \subseteq K$, we have $A \wedge B \in K$, and $A \wedge B<\mathrm{T}$, by (GEE2 $\downarrow$ ). So $B \in K \dot{\perp} \wedge B$ says

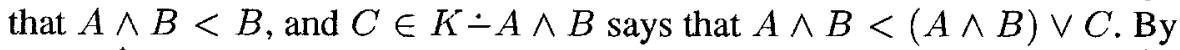
(GEE3 $\left.{ }^{\uparrow}\right)$, then, $A \wedge B<((A \wedge B) \vee C) \wedge B$, or equivalently, by (GEE2 ${ }^{\uparrow}$ ), $A \wedge B<(A \vee C) \wedge B$. Hence, by Lemma 4(iii), $A<A \vee C$. But this means that $C \in K \dot{-}$, as desired.

Now let $<$ in addition satisfy (GEE5).

$(\mathrm{K}-4)$ : Let $\nvdash A$. We need to show that $A \notin K \dot{-}$. By (Def-), we are done if $A \notin K$. So let $A \in K$. Now by (GEE5) $A<\mathrm{T}$, so by (Def-) $K \dot{-} A=K \cap\{B: A<A \vee B\}$. We are ready if we have $A \nless A \vee A$. But this follows from parts (i) and (ii) of Lemma 4.

Now let $<$ satisfy $\left(\mathrm{GEE}^{\prime}\right)$.

$(\mathrm{K}-7)$ : Let $C \in K \dot{-} A \cap K \dot{-} B$. We need to show that $C \in K \dot{-} \wedge \wedge B$. We already noted in the text that $(\mathrm{K}-7 \mathrm{p})$ implies the claim whenever $K \dot{-} \neq K$ 
and $K \dot{-} B \neq K$. So let $K \dot{-} A=K$ (the case $K \dot{-} B=K$ is similar). Then

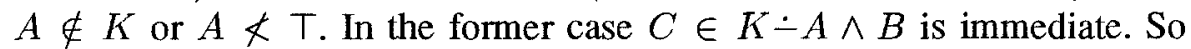
assume $A \nless \mathrm{T}$. If $K-B=K$, then $B \notin K$ or $B \nless \top$. In the former case $C \in K \dot{-A} \wedge B=K$ is immediate; in the latter case, we get $A \wedge B \nless \mathrm{T}$, as a consequence of (GEE5'), so $K \dot{-} \wedge \wedge=K$ again follows, and the claim is verified. If $K \dot{-} B \neq K$ then $C \in K \dot{-} B$ means that $B<B \vee C$. We have to show that $A \wedge B<(A \wedge B) \vee C$. Since $B<T$ and $A \nless T$, we get $B<A$, by $\left(\mathrm{GEE} 5^{\prime}\right)$. By this and $B<B \vee C$, (GEE3 $\left.{ }^{\uparrow}\right)$ gives us $B<A \wedge(B \vee C)$, hence, by $\left(\mathrm{GEE} 2^{\uparrow}\right)$ and $\left(\mathrm{GEE} 2^{\downarrow}\right), A \wedge B<(A \wedge B) \vee C$.

(ii) Let the contraction function - over a theory $K$ satisfy $(\mathrm{K}-1)-(\mathrm{K} \dot{-})$, $(\mathrm{K} \dot{-}),(\mathrm{K} \dot{-}),(\mathrm{K}-7 \mathrm{p}),(\mathrm{K}-7 \mathrm{c})$ and $(\mathrm{K} \dot{-} 8 \mathrm{c})$.

(GEE1) is obvious from (Def $<$ ).

$\left(\mathrm{GEE}^{\uparrow}\right)$ : Let $A<H \neq \emptyset$ and $H \vdash B$. So there are $B_{1}, \ldots, B_{n}$ such that $B_{1}, \ldots, B_{n} \vdash B$ and $A<B_{i}$ for $i=1, \ldots, n$, i.e., $B_{i} \in K \dot{-A \wedge} \wedge B_{i}$ and $A \notin K \dot{-} \wedge \wedge B_{i}$, for every $i=1, \ldots, n$. We want to show that $B \in K \dot{\triangle} \wedge \wedge B$ and $A \notin K \dot{-A} \wedge B$. If $K \dot{-A} \wedge B_{i}=K$ for some $i$, then, by $A \notin K \dot{-} A \wedge B_{i}$, $A \notin K$. Hence $A \wedge B_{j} \notin K$ and, by $(\mathrm{K}-3), K \dot{-} A \wedge B_{j}=K$ for every $j$, so $B_{j} \in K$ for every $j$, so $B \in K$, by $(\mathrm{K}-1)$. Also $A \wedge B \notin K$, so

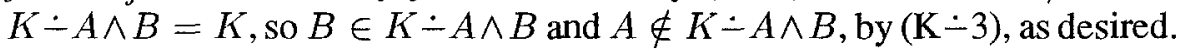
Now let $K \dot{-} A \wedge B_{i} \neq K$ for every $i$. From $B_{i} \in K \dot{-} \wedge \wedge B_{i}$, we conclude by $(\mathrm{K}-7 \mathrm{p})$ that $B_{i} \in K \dot{-} \wedge \wedge B \wedge B_{1} \wedge \ldots \wedge B_{n}$, for every $i$, so by $(\mathrm{K}-1)$ $B_{1} \wedge \ldots \wedge B_{n} \in K \dot{-} A \wedge B \wedge B_{1} \wedge \ldots \wedge B_{n}$ and $B \in K \dot{-} A \wedge B \wedge B_{1} \wedge \ldots \wedge B_{n}$. By $(\mathrm{K} \dot{-} 8 \mathrm{c}), K \dot{-} A \wedge B \wedge B_{1} \wedge \ldots \wedge B_{n} \subseteq K \dot{-} A \wedge B$, so $B \in K \dot{-} A \wedge B$,

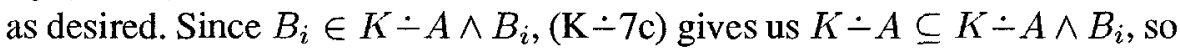

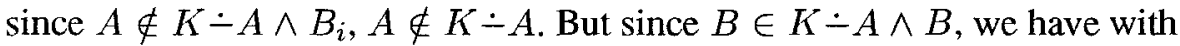
$(\mathrm{K} \dot{-} 8 \mathrm{c})$ that $K \dot{-} A \wedge B \subseteq K \dot{-}$. So $A \notin K \dot{-} A \wedge B$, as desired.

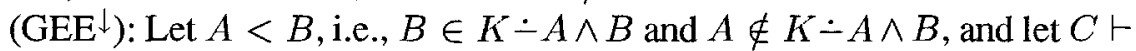

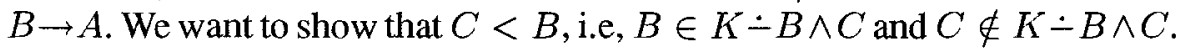
By $(\mathrm{K}-6)$, we have $K \dot{-} B \wedge C=K \dot{-}(B \wedge C \wedge(B \rightarrow A))=K \dot{-} A \wedge B \wedge C$,

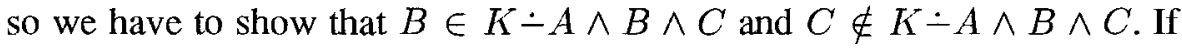
$K \dot{-} A \wedge B=K$ then $A \notin K$, since $A \notin K \dot{-} \wedge \wedge B$, so $A \wedge B \wedge C \notin K$,

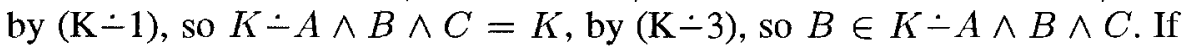

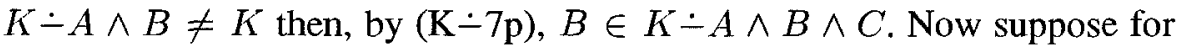
reductio that also $C \in K \dot{-} \wedge \wedge \wedge C$. By $(\mathrm{K} \dot{-} 8 \mathrm{c})$, then $C \in K \dot{-} \wedge \wedge B$, so by $C \vdash B \rightarrow A$ and $(\mathrm{K} \dot{-} 1), B \rightarrow A \in K \dot{ } \wedge \wedge B$, so by $B \in K \dot{-} \wedge \wedge B$ and $(\mathrm{K}-1)$ again, $A \in K \dot{-A} \wedge B$, contradicting our hypothesis.

(GEE4): Let $K \neq L$. Since $K$ is a theory, $A \wedge \perp \notin K$. So $K \dot{-} A \wedge \perp=K$, by (K-3). But then clearly $\perp<A$, i.e., $A \in K \dot{-} A \wedge \perp$ and $\perp \notin K \dot{-} A \wedge \perp$, if and only if $A \in K$.

(GEE4'): Let $A \in K$ and $B \notin K$. We have to show that $A \in K \dot{-A \wedge B}$ and $B \notin K \dot{-A} \wedge B$. But this is immediate from $(\mathrm{K} \dot{-} 1)$ and $(\mathrm{K} \dot{-})$. 
Now let - over $K$ in addition satisfy $(\mathrm{K}-4)$.

(GEE5): Let $\forall A$. We want to show that $A<T$. By (Def $<$ ), this means that $\top \in K \dot{-}(A \wedge \top)$ and $A \notin K \dot{-}(A \wedge \top)$. The former follows from $(\mathrm{K} \dot{-} 1)$. The latter means, by $(\mathrm{K}-6)$, that $A \notin K \dot{-}$, which follows from $\forall \forall A$ and $(\mathrm{K}-4)$.

Now let $\dot{-}$ over $K$ satisfy $(\mathrm{K} \dot{-} 7)$.

(GEE5 $5^{\prime}$ ): Let $A<\top$ and $B \nless \top$, i.e., $\top \in K \dot{-} A \wedge \top, A \notin K \dot{-} A \wedge \mathrm{T}$, and $\mathrm{T} \notin K \dot{-} B \wedge \top$ or $B \in K \dot{-} B \wedge T$. Since by $(\mathrm{K} \dot{-}) \mathrm{T}) \in K \dot{-} A \wedge \top$ and $T \in K \dot{-} B \wedge \mathrm{T}$, this means that $A \notin K \dot{-} A$ and $B \in K \dot{-} B$, by $(\mathrm{K}-6)$. We want to show that $A<B$, i.e., that $B \in K \dot{-} A \wedge B$ and $A \notin$ $K \dot{-} \wedge \wedge B$. Since $B \in K$, by $(\mathrm{K}-2)$, we have, using $(\mathrm{K}-5),(B \rightarrow A) \rightarrow B \in$ $K \dot{-}(B \rightarrow A)$, so $B \in K \dot{-}(B \rightarrow A)$, by $(\mathrm{K} \dot{-1})$. Using (K-7), we get $B \in$ $K \dot{-B} \cap K \dot{-}(B \rightarrow A) \subseteq K \dot{-}(B \wedge(B \rightarrow A))=K \dot{-} \wedge \wedge B$, by $(\mathrm{K} \dot{-6})$. Now suppose for reductio that also $A \in K \dot{-} \wedge \wedge$. By $B \in K \dot{-} A \wedge B$ and $(\mathrm{K}-8 \mathrm{c})$,

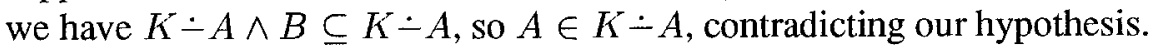

(iii) Let $<$ satisfy $(\mathrm{GEE} 1)-\left(\mathrm{GEE} 3^{\downarrow}\right)$ and let $-=\mathrm{C}(<)$ and $<^{*}=\mathrm{E}(\mathrm{C}(<))$. Then we have

$A<* B$

iff

$B \in K \dot{-} A \wedge B$ and $A \notin K \dot{-} A \wedge B$

(by $(\operatorname{Def}<)$ )

iff either $B \in K \dot{-} A \wedge B$ and $A \notin K \dot{-} \wedge \wedge$, and $A \wedge B \notin K$ or $A \wedge B \nless \top$

or $B \in K \dot{-} A \wedge B$ and $A \notin K \dot{-} A \wedge B$ and $A \wedge B \in K$ and $A \wedge B<\top$

iff either $\quad B \in K$ and $A \notin K$, and $A \wedge B \notin K$ or $A \wedge B \nless \top$

or $B \in K$ and $A \wedge B<(A \wedge B) \vee B$ and $(A \notin K$ or $A \wedge B \nless$ $(A \wedge B) \vee A)$ and $A \wedge B \in K$ and $A \wedge B<T$ (by (Def- $)$ )

iff either $A \notin K$ and $B \in K$

( $K$ is a theory)

or $A \wedge B \in K$ and $A \wedge B<B$

( $K$ is a theory, $\left(\mathrm{GEE}^{\uparrow}\right)$, Lemma 4(ii))

iff either $A \notin K$ and $B \in K$

$$
\text { or } A \wedge B \in K \text { and } A<B
$$

$\left((\mathrm{GEE} 2 \downarrow)\right.$ and $\left.\left(\mathrm{GEE} 3{ }^{\downarrow}\right)\right)$

iff

$B \in K$ and $(A \notin K$ or $A<B)$

( $K$ is a theory).

It follows readily that $<^{*}$ and $<$ agree within $K$, and that $<^{*}$ and $<$ are identical, if $<$ satisfies in addition (GEE4) and (GEE4').

(iv) Let $\dot{-}$ satisfy $(\mathrm{K} \dot{-1})-(\mathrm{K} \dot{-}),(\mathrm{K} \dot{-5})$ and $(\mathrm{K} \dot{-} 6)$, and let $<=\mathbf{E}(\dot{-})$ and $\dot{-}^{*}=\mathbf{C}(\mathbf{E}(\dot{-}))$. Then we have 
$B \in K \dot{*} * A$

iff either $B \in K$, and $A \notin K$ or $A \nless \top$

or $B \in K$ and $A<A \vee B$ and $A \in K$ and $A<T$ (by (Def $\dot{-}$ ))

iff either $B \in K$, and $A \notin K$ or $\top \notin K \dot{-} A \wedge \top$ or $A \in K \dot{-A \wedge \top}$

or $A, B \in K$ and $\top \in K \dot{-} A \wedge \top$ and $A \notin K \dot{-} A \wedge \top$ and $A \vee B \in K \dot{-}(A \wedge(A \vee B))$ and $A \notin K \dot{-}(A \wedge(A \vee B))$

(by $(\operatorname{Def}<)$ )

iff either $B \in K$, and $A \notin K$ or $A \in K \dot{-} A$

or $\quad A, B \in K$ and $A \notin K \dot{-} A$ and $A \vee B \in K \dot{-} A \quad$ (by $(\mathrm{K} \dot{-1})$ and $(\mathrm{K} \dot{-} 6))$

iff either $B \in K$ and $K \dot{-}=K \quad$ (by $(\mathrm{K} \dot{-} 1)-(\mathrm{K} \dot{-})$ and $(\mathrm{K} \dot{-} 5)$ )

or $B \in K$ and $A \in K$ and $A \notin K \dot{-} A$ and $B \in K \dot{-} A$

(by $(\mathrm{K} \dot{-} 5)$ and $(\mathrm{K} \dot{-} 1))$

iff either $B \in K \dot{-} A$ and $K \dot{-} A=K$

or $B \in K \dot{-}$ and $K \dot{-} \neq \neq \neq$ (by $(\mathrm{K} \dot{-} 1)-(\mathrm{K} \dot{-} 3)$ and $(\mathrm{K}-5))$

iff $B \in K \dot{\circ}$.

Proof of Theorem 3 Let $R$ be a binary relation over $L$. Clearly, if $R$ is extensible to a GEE-relation $<$, then it must satisfy (EC). For suppose there is a finite set $\left\{\left\langle A_{1}, B_{1}\right\rangle, \ldots,\left\langle A_{n}, B_{n}\right\rangle\right\} \subseteq R \subseteq<$ such that $B_{1} \wedge \ldots \wedge B_{n} \vdash$ $A_{1} \wedge \ldots \wedge A_{n}$. Then Lemma 4(iv) and (GEE2 ${ }^{\uparrow}$ ) give us $A_{1} \wedge \ldots \wedge A_{n}<$ $A_{1} \wedge \ldots \wedge A_{n}$, contradicting Lemma 4(ii).

For the converse direction, suppose that $R$ satisfies (EC). Now define two operators $U p$ and Down on binary relations $P$ over $L$ as follows:

$U p(P)=\left\{\langle A, B\rangle:\right.$ there are pairs $\left\langle A, C_{1}\right\rangle, \ldots,\left\langle A, C_{m}\right\rangle \in P$ such that $\left.C_{1}, \ldots, C_{m} \vdash B\right\}$

$\operatorname{Down}(P)=\{\langle A, B\rangle:$ there is a pair $\langle C, B\rangle \in P$ such that $A \vdash B \rightarrow C\}$

Then define $R_{0}=R, R_{2 i+1}=U p\left(R_{2 i}\right), R_{2 i+2}=\operatorname{Down}\left(R_{2 i+1}\right)$, and $<=\bigcup_{i \in \omega} R_{i}$.

Now clearly, $R \subseteq<$, since $R_{i} \subseteq R_{i+1}$ for every $i$. Moreover, < satisfies $\left(\mathrm{GEE}^{\uparrow}\right)$ and $\left(\mathrm{GEE}^{\downarrow}\right)$, by construction and the finitary character of $\left(\mathrm{GEE}^{\uparrow}\right)$. Finally, for (GEE1), suppose for reductio that $T<T$. Then $\langle T, T\rangle \in R_{i}$ for some $i$. This $R_{i}$ then violates (EC). But as $R$ satisfies (EC), this contradicts the fact that both $U p$ and Down preserve (EC), as we are now going to show. 
First, suppose that a relation $P$ satisfies (EC), but $U p(P)$ does not. The latter means that there is a finite set $\left\{\left\langle A_{1}, B_{1}\right\rangle, \ldots,\left\langle A_{n}, B_{n}\right\rangle\right\} \subseteq U p(P)$ such that $B_{1} \wedge \ldots \wedge B_{n} \vdash A_{1} \wedge \ldots \wedge A_{n}$. By the definition of $U p(P)$, then, there are finite sets $H_{1}, \ldots, H_{n}$ such that $H_{i} \vdash B_{i}$ and $\left\langle A_{i}, C\right\rangle \in P$ for every $C \in H_{i}$. But since $\wedge H_{1} \wedge \ldots \wedge \wedge H_{n} \vdash A_{1} \wedge \ldots \wedge A_{n}$, we have a violation of (EC) in $P$, contradicting the assumption that $P$ satisfies (EC).

Second, suppose that a relation $P$ satisfies (EC), but $\operatorname{Down}(P)$ does not. The latter means that there is a finite set $\left\{\left\langle A_{1}, B_{1}\right\rangle, \ldots,\left\langle A_{n}, B_{n}\right\rangle\right\} \subseteq$ $\operatorname{Down}(P)$ such that $B_{1} \wedge \ldots \wedge B_{n} \vdash A_{1} \wedge \ldots \wedge A_{n}$. By the definition of $\operatorname{Down}(P)$, then, there are sentences $C_{1}, \ldots, C_{n}$ such that $A_{i} \vdash B_{i} \rightarrow C_{i}$ and $\left\langle C_{i}, B_{i}\right\rangle \in P$ for every $i$. But then $B_{1} \wedge \ldots \wedge B_{n} \vdash\left(B_{1} \rightarrow C_{1}\right) \wedge \ldots \wedge\left(B_{n} \rightarrow C_{n}\right)$, and hence $B_{1} \wedge \ldots \wedge B_{n} \vdash C_{1} \wedge \ldots \wedge C_{n}$, contradicting the assumption that $P$ satisfies (EC).

This completes the proof of the theorem. (Note incidentally that $<$ as constructed above is the smallest GEE-relation extending $R$.)

Proof of Lemma 6 Clearly, a pair $\langle A, B\rangle$ is inconsistent with a GEErelation $<$ if $B<B \rightarrow A$. For every GEE-relation with $B<B \rightarrow A$ and $A<$ $B$ would have $B \wedge A<((B \rightarrow A) \wedge B)$, by Lemma 4(iv), so $B \wedge A<B \wedge A$, by $($ GEE $2 \uparrow)$, contradicting Lemma 4(ii).

Now suppose for the converse that $\langle A, B\rangle$ is inconsistent with $<$. Since $<$ is a GEE-relation, it satisfies (EC). Since $\langle\cup\langle A, B\rangle$ is inconsistent, however, it does not satisfy (EC). Hence there are $D_{1}, \ldots, D_{n}, E_{1}, \ldots, E_{n}$ such that $D_{i}<E_{i}$ for every $i$ and $E_{1} \wedge \ldots \wedge E_{n} \wedge B \vdash D_{1} \wedge \ldots \wedge D_{n} \wedge A$. Now application of Lemma 4(iv) gives us $D_{1} \wedge \ldots \wedge D_{n}<E_{1} \wedge \ldots \wedge E_{n}$. Hence, by $\left(\mathrm{GEE}^{\downarrow}\right),\left(\left(E_{1} \wedge \ldots \wedge E_{n}\right) \rightarrow\left(D_{1} \wedge \ldots \wedge D_{n}\right)\right)<E_{1} \wedge \ldots \wedge E_{n}$. But on the one hand, $B \vdash\left(E_{1} \wedge \ldots \wedge E_{n}\right) \rightarrow\left(D_{1} \wedge \ldots \wedge D_{n}\right)$. So by (GEE2 $\downarrow$ ), $B<E_{1} \wedge \ldots \wedge E_{n}$. And on the other hand, $E_{1} \wedge \ldots \wedge E_{n} \vdash B \rightarrow A$. So by $(\mathrm{GEE} 2 \uparrow), B<B \rightarrow A$, as desired.

Proof of Lemma 7 We decided to set aside (SEE4) and (SEE5). By Lemma 5, then, it suffices to show that $<$ is extensible to a GEE-relation which satisfies virtual connectivity, (SEE1).

Let $<$ be a GEE-relation. < satisfies (EC). Since $L$ is supposed to be countable, we can fix an enumeration $\left\langle A_{1}, B_{1}, C_{1}\right\rangle,\left\langle A_{2}, B_{2}, C_{2}\right\rangle,\left\langle A_{3}, B_{3}, C_{3}\right\rangle, \ldots$ of all triples in $L$. We make true all instances of virtual connectivity by working through this list in the following manner. If $A_{i} \nless B_{i}$, we step to the next triple. If $A_{i}<B_{i}$, on the other hand, we check whether one of $A_{i}<C_{i}$ and $C_{i}<B_{i}$ holds. If so, we again step to the next triple. If not, we add one of the pairs $\left\langle A_{i}, C_{i}\right\rangle$ and $\left\langle C_{i}, B_{i}\right\rangle$ to $<$, and we do this in such a way that (EC) is preserved. We shall presently show that this is possible. Let the new pair be $\left\langle A_{i}, C_{i}\right\rangle$; the other case is similar. Then we extend $\left\langle\cup\left\langle A_{i}, C_{i}\right\rangle\right.$ to a new GEE-relation, which is possible, by Theorem 3 . We repeat that process for 
every $i \in \omega$. Then we define $<^{+}$as the union of all these GEE-relations. Since $<^{+}$is the union of a non-empty chain of GEE-relations, it is itself a GEErelation. And it satisfies (SEE1) by construction. So $<^{+}$is a SEE-relation extending $<$, i.e., $<\subseteq<^{+}$, as desired.

We are left to show that it is possible consistently to extend a GEE-relation $<$ such that $A<B$ by either $\langle A, C\rangle$ or $\langle C, B\rangle$. Suppose it is not. Then, by Lemma 6, we have $C<C \rightarrow A$ and $B<B \rightarrow C$. But also $A<B$. Putting these three pieces together with the help of Lemma 4(iv), we get $C \wedge B \wedge A<$ $(C \rightarrow A) \wedge(B \rightarrow C) \wedge B$. By $\left(\right.$ GEE2 $\left.{ }^{\uparrow}\right)$, we finally get $C \wedge B \wedge A<C \wedge B \wedge A$, contradicting Lemma 4(ii). This completes the proof of the lemma.

Proof of Theorem 4 Clearly, every GEE-relation < is a subset of the meet of all SEE-relations extending it. To show that it is no proper subset, suppose that $A \nless B$. We have to show that there is a SEE-relation $<^{+}$ extending < such that $A \nless^{+} B$. To see that this is true, we first add, under preservation of (EC), $\langle B, B \rightarrow A\rangle$ to $<$. We shall presently prove that this is possible. Then we extend this set to a SEE-relation $<^{+}$, which is possible according to Lemma 8 . But by Lemma 6 , no GEE-relation, and a fortiori no SEE-relation, containing $\langle B, B \rightarrow A\rangle$ can contain $\langle A, B\rangle$. Thus we have found a SEE-relation $<^{+}$extending $<$such that $A \nless^{+} B$, as desired.

We are left to show that it is possible consistently to extend a GEE-relation $<$ such that $A \nless B$ by the pair $\langle B, B \rightarrow A\rangle$. Suppose it is not. Then, by Lemma 6 , we have $B \rightarrow A<(B \rightarrow A) \rightarrow B$. This gives us, by (GEE2 ${ }^{\uparrow}$ ), $B \rightarrow A<B$. Hence by $\left(\mathrm{GEE} 2^{\downarrow}\right), A<B$, contradicting our hypothesis. This completes the proof.

\section{REFERENCES}

Alchourrón, C., Gärdenfors, P., and Makinson, D., 1985, "On the logic of theory change: partial meet contraction and revision functions," Journal of Symbolic Logic 50, 510-530.

Alchourrón, C. and Makinson, D., 1985, "On the logic of theory change: safe contraction," Studia Logica 44, 405-422.

Alchourrón, C. and Makinson, D., 1986, "Maps between some different kinds of contraction function: the finite case," Studia Logica 45, 187-198.

Fuhrmann, A., 1988, Relevant Logics, Modal Logics, and Theory Change, $\mathrm{PhD}$ thesis, Australian National University, Canberra.

Gärdenfors, P., 1988, Knowledge in Flux: Modeling the Dynamics of Epistemic States, Bradford Books, MIT Press, Cambridge, Mass.

Gärdenfors, P. and Makinson, D., 1988, "Revisions of knowledge systems using epistemic entrenchment." pp. 83-95 in Theoretical Aspects of Reasoning About Knowledge, M. Vardi, ed., Los Altos, CA: Morgan Kaufmann.

Ginsberg, M., 1986, "Counterfactuals," Artificial Intelligence 30, 35-79.

Herzberger, H., 1973, "Ordinal preference and rational choice," Econometrica 41, 187-237.

Katsuno, H. and Mendelsson, A., 1992, "On the difference between updating a knowledge base and revising it." forthcoming in Belief Revision, P. Gärdenfors, ed., Cambridge: Cambridge University Press. 
Kratzer, A., 1981, "Partition and revision: the semantics of counterfactuals," Journal of Philosophical Logic 10, 201-216.

Kraus, S., Lehmann, D., and Magidor, M., 1990, "Nonmonotonic reasoning, preferential models and cumulative logics," Artificial Intelligence 44, 167-207.

Lehmann, D. and Magidor, M., 1990, "What does a conditional knowledge base entail?," Leibniz Center for Research in Computer Science, Hebrew University of Jerusalem, TR-90-10 (to appear in Artificial Intelligence).

Lewis, D., 1981, "Ordering semantics and premise semantics for counterfactuals," Journal of Philosophical Logic 10, 217-234.

Lindström, S. and Rabinowicz, W., 1991, "Epistemic entrenchment with incomparabilities and relational belief revision." pp. 93-126 in The Logic of Theory Change, Lecture Notes in Computer Science, Vol. 465, A. Fuhrmann and M. Morreau, eds., Berlin: Springer.

Makinson, D., 1989, "General theory of cumulative inference." pp. 1-18 in Non-Monotonic Reasoning - Proceedings of the 2nd International Workshop 1988, M. Reinfrank et al., eds., Berlin: Springer.

Makinson, D., 1990, "General patterns in nonmonotonic reasoning." forthcoming in Handbook of Logic in Artificial Intelligence and Logic Programming, Vol. II: Nonmonotonic and Uncertain Reasoning, D.M. Gabbay et al., eds., Oxford: Oxford UP.

Makinson, D. and Gärdenfors, P., 1991, "Relations between the logic of theory change and nonmonotonic logic." pp. 185-205 in The Logic of Theory Change, Lecture Notes in Computer Science, Vol. 465, A. Fuhrmann and M. Morreau, eds., Berlin: Springer.

Morreau, M. and Rott, H., 1991, "Is it impossible to keep up to date?" pp. 233-243 in Nonmonotonic and Inductive Logic - Proceedings of the Ist International Workshop 1990, Lecture Notes in Computer Science, Vol. 543, J. Dix, K. P. Jantke and P. H. Schmitt, eds., Berlin: Springer.

Nebel, B., 1989, "A knowledge level analysis of belief revision." pp. 301-311 in Proceedings of the 1st International Conference on Principles of Knowledge Representation and Reasoning, R. Brachman, H. Levesque and R. Reiter, eds., San Mateo, CA: Morgan Kaufmann.

Rott, H., 1991a, "Two methods of constructing contractions and revisions of knowledge systems," Journal of Philosophical Logic 20, 149-173.

Rott, H., 1991b, "A nonmonotonic conditional logic for belief revision I." pp. 135-183 in The Logic of Theory Change, A. Fuhrmann and M. Morreau, eds., Lecture Notes in Computer Science, Vol. 465, Berlin: Springer.

Rott, H., 1992, "On the logic of theory change: more maps between different kinds of contraction function." forthcoming in Belief Revision, P. Gärdenfors, ed., Cambridge: Cambridge University Press.

Sen, A., 1982, Choice, Welfare and Measurement, Oxford: Blackwell.

Veltman, F., 1976, "Prejudices, presuppositions and the theory of counterfactuals." pp. 248281 in Amsterdam Papers of Formal Grammar, Vol. I, J. Groenendijk and M. Stokhof, eds., Amsterdam: Centrale Interfaculteit, Universiteit Amsterdam. 